\title{
Circumstellar discs: What will be next?
}

\author{
Quentin Kral, Cathie Clarke and Mark Wyatt
}

\begin{abstract}
This prospective chapter gives our view on the evolution of the study of circumstellar discs within the next 20 years from both observational and theoretical sides. We first present the expected improvements in our knowledge of protoplanetary discs as for their masses, sizes, chemistry, the presence of planets as well as the evolutionary processes shaping these discs. We then explore the older debris disc stage and explain what will be learnt concerning their birth, the intrinsic links between these discs and planets, the hot dust and the gas detected around main sequence stars as well as discs around white dwarfs.
\end{abstract}

\section{Protoplanetary discs}

Protoplanetary discs are the discs of gas and dust that surround a significant fraction of stars with ages less than a few - 10 Myr. The best studied examples are located in nearby star forming clouds (typically around $150 \mathrm{pc}$ from the Earth) but evidence of discs is found in more distant clusters and even in star forming regions in the Magellanic Clouds. Protoplanetary discs are often termed 'primordial discs' in order to indicate that they are composed of material from the local interstellar medium which has collapsed into a centrifugally supported disc around the young star. Their typical gas and dust inventories (tens of Jupiter masses and tens to hundreds of Earth masses respectively) imply that at this stage they have sufficient material to form (exo-)planetary systems. Nevertheless, it is clear that not all the material in protoplanetary discs is destined to turn into planets. There is good evidence that there are significant accretion flows from the discs onto their central stars while winds,

Institute of Astronomy, University of Cambridge, Madingley Road, Cambridge CB3 0HA, UK, e-mail: qkraleast.cam.ac.uk, cclarkedast.cam.ac.uk 
either magnetohydrodynamical or thermal, are likely to play a role in dispersing such discs after a few Myr.

\section{The properties of protoplanetary discs}

\section{Disc masses}

Currently there is considerable uncertainty about the robustness of the techniques used to assess disc gas mass. In default of better indicators, the traditional approach has been to assess dust mass from the mm continuum flux and convert to a total gas mass by assuming a dust to gas ratio of 1:100 as in the interstellar medium. This is a questionable assumption since there are a variety of processes that can drive differential evolution of dust and gas within discs. Direct gas measurements are however hard: since the infrared lines of molecular hydrogen (the dominant gas species) are weak and do not trace the bulk of the gas mass (e.g. Pascucci et al. 2013), proxy molecules, particularly $\mathrm{CO}$, are used instead. Although the most abundant isotopologue of $\mathrm{CO}\left({ }^{12} \mathrm{CO}\right)$ cannot be used as a mass tracer because it is usually optically thick, the high sensitivity of ALMA permits measurements of the rarer isotopologues, ${ }^{13} \mathrm{CO}$ and $\mathrm{C}^{18} \mathrm{O}$ (Williams \& Best 2014) and these are converted into total gas mass assuming an abundance equal to that in the dense interstellar medium (Lacy et al. 1994). CO based total gas masses are however much lower than those obtained from dust measurements and, when combined with measurements of accretion onto the central star, can imply disc lifetimes much less than the system age (Manara et al. 2016). While the problem can be partially mitigated by careful treatment of photodissociation and freeze-out of CO (e.g. Miotello et al. 2014), the low values of $\mathrm{CO}$ based gas mass estimates have been challenged by HD 1-0 line measurements from Herschel (Bergin et al. 2013) which imply gas masses that are a factor 3 to 100 higher than those based on CO. Current modelling (Trapman et al. 2017) suggests that carbon depletion is the most likely source of this discrepancy.

This uncertainty about disc gas masses implies uncertainties about the environment of forming protoplanets and even the planet formation mechanism. Disc mass is a crucial discriminant between the two main competing models for gas giant planet formation (i.e. core accretion versus gravitational instability; Lissauer \& Stevenson 2007, Durisen et al. 2007), since the latter requires disc masses that are in the region of $10 \%$ of the central stellar mass. High resolution imaging (e.g. in the submm continuum with ALMA) can however provide an alternative tool for assessing whether the conditions for gravitational instabilty are in fact being met (see Tobin et al. 2016 for evidence of a disc undergoing gravitational fragmentation, albeit on a stellar rather than planetary scale and Perez et al. 2016)(Fig. 11) for disc spiral structure which may be interpreted as evidence for gravitational instability in the disc).

Ultimately, the issue of disc gas mass will not be settled definitively until HD 1-0 measurements become available for a broader range of systems and even then their 


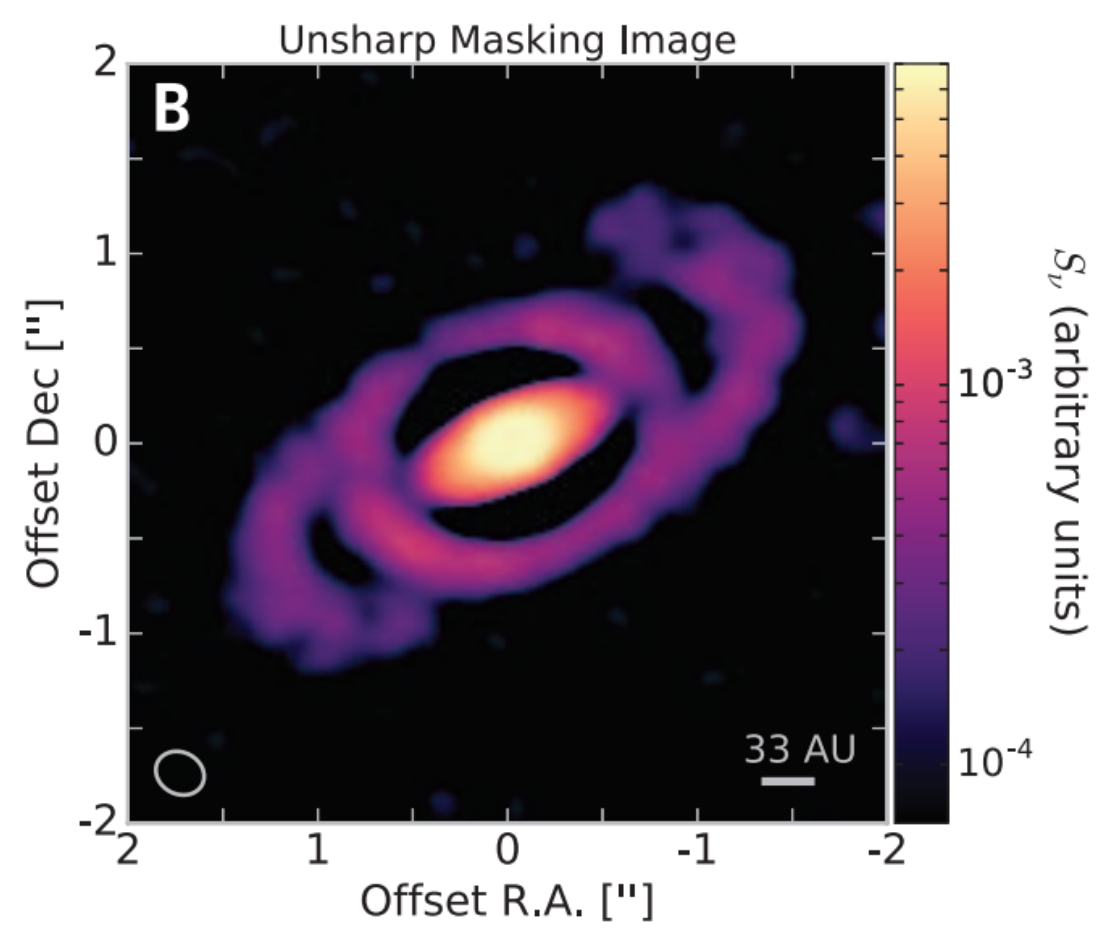

Fig. 1 An example of spiral structure on a scale of 100-200au in the young star Elias 227 obtained by applying unsharp masking to a high resolution ALMA continuum image (Perez et al. 2016).

interpretation will need a sophisticated modelling effort combined with information from high resolution mapping in other tracers such as CO. From 2018, further HD measurements will become available using the HIRMES instrument aboard SOFIA (Trapman et al. 2017), while there are more distant possibilities of achieving still higher sensitivity via the proposed SAFARI instrument on SPICA (Roelfsema et al. |2014).

\section{Disc radii}

In recent years, it has become possible to measure disc outer radii in both gas ( $\mathrm{CO}$ lines) and dust (generally mm continuum). The increased spatial resolution and sensitivity of $\mathrm{mm}$ arrays has allowed surveys to sample the diversity of protoplanetary disc sizes: while the largest discs extend over many hundreds of au (Guilloteau et al. 2011. 2013) there are other systems for which the spectral energy distribution as measured by Herschel implies dust only within an au of the star (Bulger et al. |2014). There is also a general trend for discs to be more compact in dust than in gas (Panic et al. 2009, Pietu et al. 2014), a result that can be readily understood 
in terms of inward radial migration of dust (Birnstiel \& Andrews 2014). Indeed it has recently become apparent (e.g. Pietu et al. 2014) that discs that are faint in the mm continuum (which had previously been interpreted as having low gas mass) may instead be merely compact (and thus optically thick in the dust continuum).

Currently there has been little attempt to fit the growing dataset of disc outer radii into a protoplanetary disc evolutionary scenario. Outer disc radii are important to planet formation for a number of reasons. First of all, for typical surface density profiles (Andrews \& Williams 2005), the bulk of disc mass is contained at large radius, even if planets only form in the inner regions of the disc, their natal environments are probably re-supplied by flows of gas and solids from large radii. Outer disc radii also indicate the processes shaping disc evolution, which are important for the migration of protoplanets in the disc. Protoplanetary discs are often modelled as conventional viscous accretion discs in which radial inflow in the disc is driven by redistribution of angular momentum to large disc radii resulting in an increase in outer disc radius with time (e.g. Hartmann et al. 1998). On the other hand, recent modelling suggests that the dominant evolutionary driver may instead be large scale magnetohydrodynamical winds (Bai 2013; Simon et al. 2013) which, in removing angular momentum from the disc, may instead cause the secular decrease of disc radius with time. A further complication is that even the rather mild ultraviolet radiation fields in star forming regions are likely to drive significant winds from the outer regions of discs by photoevaporation (e.g. Facchini et al. 2016) which should cause shrinkage of disc gas at late times (Clarke 2007; Anderson et al. 2013).

\section{Grain growth}

An area of recent progress has been the quantification of grain growth in protoplanetary discs. In the core accretion scenario it is a fundamental tenet that grains grow from the sub-micron scales of dust in the interstellar medium to scales where they ultimately coalesce into planetary cores. While it is still unclear whether this involves direct growth to planetesimal ( $\mathrm{km}$ scale) bodies or instead the accretion of (roughly cm scale) 'pebbles' (Lambrechts \& Johansen 2012), both variants imply that discs should evidence grain growth. Such evidence has been widely available through compilation of submm spectral indices which are sensitive to the maximum grain size in the dust population: if dust grows such that the size scales dominating the cross section are larger than the wavelength of observation, the opacity becomes 'grey' and thus the spectral slope of optically thin emission simply follows the Rayleigh Jeans dependence on wavelength. Steeper spectral indices (more flux at shorter wavelengths) however imply frequency dependent opacity and hence more compact grains. While indications of grey opacity (and hence grain growth) in unresolved observations of protoplanetary discs have been available for over a decade (Ricci et al. 2010; Testi et al. 2014), it is only with the superior resolution of ALMA that it has become possible to conduct this experiment as a function of radius within individual discs (Tazzari et al. 2016). Pilot studies provide strong evidence that the maximum grain size declines with increasing radius. It is currently unclear whether 
this indicates more efficient growth of grains in situ in the inner regions of discs or whether the larger grains in the outer disc have already become decoupled from the more tenuous gas at large radius and undergone rapid inward radial migration (e.g. Birnstiel \& Andrews 2014). Whatever the evolutionary interpretation of this data, it is evident that ALMA has the capability to address this issue in much larger samples in the coming years and that this will provide good constraints on the solid material available to planet formation at various disc radii. It is however worth noting that this method cannot be applied in the innermost disc - less than about 10 au - due both to finite resolution and the impossibility of applying this technique in regions of the disc that are optically thick in the mm continuum. Finally, although ALMA promises a spectacular increase in our understanding of grain growth to $\mathrm{mm}$ scales, it will be necessary to go to longer wavelengths in order to constrain grain growth to larger $(>\mathrm{cm})$ scales: ngVLA and the higher frequency bands of SKA offer the prospect of probing such large grains in the coming decade.

\section{Disc chemistry}

We now turn to the intense efforts in recent years to characterise radial variations in the chemistry of protoplanetary discs. Here again, increases in resolution and sensitivity will facilitate the assessment of chemical conditions as a function of radius (rather than simply obtaining disc averaged quantities that are difficult to interpret). An area that is currently in its infancy, due to the difficulties of removing contaminating envelope emission, is the chemical characterisation of the youngest, most deeply embedded discs (i.e. Class 0/I sources: see below). Here, new theoretical studies are charting the chemical processing of cloud abundance patterns that are to be expected during the early phases of disc assembly (Drozdovskaya et al. 2015), as well as possible chemical signatures of shock heating in self-gravitating discs (Ilee et al. 2011). Such studies can be confronted with ALMA data (Douglas et al. 2013) and with compositional information on cometary ices in the solar system (e.g. Le Roy et al. 2015) and will help clarify the initial chemical conditions during the planet formation era.

Considering the large scale distributions of carbon and oxygen in discs, the main reservoirs of these elements in discs are in the form of $\mathrm{CO}, \mathrm{CO}_{2}$ and $\mathrm{H}_{2} \mathrm{O}$, with minority components in carbide and silicate grains. These species transition between their solid and gaseous phases at their respective snow-lines which are located, for typical disc model parameters in solar type stars at $\sim 30 \mathrm{au}, 5 \mathrm{au}$ and $2 \mathrm{au}$. There has been considerable interest in locating $\mathrm{CO}$ snow-lines in protoplanetary discs (as marked by the steep decline in $\mathrm{C}^{18} \mathrm{O}$ emission and the associated rise in species such as $\mathrm{DCO}^{+}$and $\mathrm{N}_{2} \mathrm{H}^{+}$(Qi et al. 2011, Mathews et al. 2013, Qi et al. 2013, 2015), partly in order to obtain an anchor point for modelling disc temperature structure, assuming that the $\mathrm{CO}$ snow-line should correspond to a local disc mid-plane temperature of around $20 \mathrm{~K}$. This exercise is however somewhat complicated by the fact that the sublimation temperature of $\mathrm{CO}$ can vary according to whether it is in the form of pure CO ice or mixed CO-water ice (Collings et al. 2004). There is neverthe- 
less the prospect that the location of $\mathrm{CO}$ snow-lines in large samples of protostellar discs will provide one of the most direct insights into the thermal evolution of the mid-plane regions of protoplanetary discs (Panic \& Min 2017).

Apart from their interest as disc thermometers, $\mathrm{CO}$ snow-lines mark the region where most of the carbon in the disc makes the transition between solid phase (CO ice) and gaseous CO. Inward of the snow-line, around 50\% of the oxygen in the disc enters the gas phase as gaseous $\mathrm{CO}$, while, outward of the water snow-line on a scale of $\sim 1 \mathrm{au}$, the remainder resides in solid form as water ice.

This division of carbon and oxygen between solid and gaseous phases at various radii provides the chemical backdrop for planet formation and may in principle allow determinations of elemental abundances in hot Jupiters to constrain planet formation models. The mapping from formation scenario to chemical signature is however complex and somewhat degenerate. While the division of carbon and oxygen into gaseous and solid forms in various thermal regimes is reasonably well understood, planet formation involves a complex interplay between the accretion of gaseous and solid phases along with planetary migration. In addition, elemental abundances are almost certainly functions of radius on account of the fact that loosely coupled icy dust grains drift inwards relative to the gas; at the same time this drift can be impeded by grain accumulation in pressure maxima and near ice-lines (Ida \& Lin 2008). Analysis of elemental abundances of material accreting onto the star (Drake et al. 2005; Ardila et al. 2013, Kama et al. 2015) provides some evidence that objects with structures where dust accumulates do show evidence for chemical filtration (i.e. a deficit of elements that are retained in the disc in the form of grains or icy grain mantles). The relationship between chemical fractionation, resolvable dust structures in discs and the chemical composition of planets is clearly an area that requires more thorough exploration (Madhusudhan et al. 2014, 2016, Booth et al. 2017).

Overall, chemical studies of protoplanetary discs will derive great benefit from the fact that the high resolution spectroscopy provided by ALMA in the submm and METIS in the mid-infrared are well matched in spatial resolution (around 50 mas). This will permit a spatial dissection of disc chemistry on scales of a few au in the closest star forming regions and will thus directly access chemistry in the planet forming regions of discs.

\section{Evidence for planets in protoplanetary discs}

There are currently relatively few examples of planets discovered in protoplanetary discs by the methods that are traditional for older planetary systems: evidently the large radial optical depth of protoplanetary discs rules out transit methods during the disc bearing phase. Radial velocity detections are impeded by false signals owing to the active surface features on young stars, whereas imaging studies need to contend with the possibility of false positives generated by scattering off clumpy disc features. The former problem can to some extent be mitigated by near-infrared 
spectroscopic monitoring, allowing starspot signals to be distinguished from planets: this has led to the first claimed detection of a radial velocity planet in a disc bearing star (CI Tau, Johns-Krull et al.|2016). At an age of only around a Myr this provides important evidence that at least some gas giant planets can form and migrate to small orbital radii at a very early stage of protoplanetary disc evolution. It is currently unclear whether the moderate eccentricity of this planet $(\sim 0.3)$ is to be understood in terms of pumping by the massive disc or whether it requires the presence of sibling planets (Rosotti et al. 2017). There have also been several claims of point source detections in protoplanetary discs which may be interpreted as planets (e.g. Huelamo et al. 2011; Kraus \& Ireland 2012; Quanz et al. 2015; Sallum et al. |2015; Thalmann et al. 2015) though none have been confirmed thus far.

The majority of information about possible planetary systems in disc bearing stars derives from the effect that such planets have upon the disc. Occasionally this is in the form of a stable periodic feature in disc spectroscopy as in the candidate hot Jupiter embedded in the vigorously accreting young stars FU Orionis (Powell et al. 2012). The majority of evidence is however derived from disc structures imaged either in scattered light (where it represents a perturbation of small grains at several scale heights above the disc) or else in submm continuum (where it instead represents structure in large grains close to the disc mid-plane).

Many but not all of the growing compendium of imaged disc structures have been found in systems that had been previously classified as 'transition discs' on account of their spectral energy distributions: these evidenced missing emission over a limited range of wavelengths, which suggested the presence of annuli or holes devoid of dust. For example TW Hydra (see Fig. 3), a system identified as a transition disc from its spectral energy distribution (Calvet et al. 2002) has recently been shown to exhibit a wealth of annular structure in near-infrared scattered light and submm imaging (van Boekel et al. 2016, Andrews et al. 2016). The term 'transition disc' reflected the belief that such discs belong to the evolutionary phase where they make the transition between optically thick protoplanetary disc status to debris disc phase (see next section). This is now thought to be not necessarily the case: many so-called transition discs are vigorously accreting and have very high mm fluxes (Owen \& Clarke 2012; Najita et al. 2015), suggesting that they instead belong to an early evolutionary stage. Modelling of planet carved structures in discs initially involved only gas (e.g. Lin \& Papaloizou 1979), where it was found that significant perturbation in the gas required rather massive planets (around a Jupiter mass or above, Crida et al. 2006). It is now well known (Paardekooper \& Mellema 2004: Rice et al. 2006: Zhu et al. 2012; Owen 2014; Zhu et al. 2014; Dong et al. 2015: Picogna \& Kley 2015) that observable structures in dust can be produced by much lower mass planets which hardly perturb the gas. For planets more massive than 20 Earth masses, drag coupled dust is trapped in the pressure maximum in the disc just outward of the planet and this trapping is predicted to lead to a hole in the $\mathrm{mm}$ dust distribution. Lower mass planets produce an annular dust feature outside the planet's orbital radius but no interior hole. Simulations (Rosotti et al. 2016) suggest that dusty structures in discs produced by low mass planets should be detectable both in the submm continnum with ALMA and in the infrared using either existing 
instruments telescopes such as SPHERE on the VLT or GPI on Gemini or else in the thermal mid-infrared using future intruments such as METIS on the E-ELT. The limits of detectability (around 15 Earth masses) are expected to be achieved with ALMA.

Apart from the annular structures discussed above, several transition discs exhibit spiral structure in near-infrared scattered light imaging (Muto et al. 2012; Garufi et al. 2013; van der Marel et al. 2013; Grady et al. 2013; Christiaens et al. 2014, Benisty et al. 2015; Wagner et al. 2015, Garufi et al. 2016; Stolker et al. |2016). Although such spirals have been attributed to the presence of planets, the interpretation is not straightforward (Juhasz et al. 2015) since the amplitude of surface density variations produced by planets (typically several tens of per cent at most) is much less than the amplitude of variation observed (a factor three or more). Planets may be able to produce the larger amplitude spirals seen in scattered light through perturbation of the disc vertical scale height, but in this case the amplitude of spiral structure in the submm is predicted to be much smaller.

It can be expected that the next decade will see a concerted attempt to compare spirals seen in scattered light and mm continuum and to use these to try and constrain the presence of planets in the disc. The largely null results that have emerged from planet searches in somewhat older (10-300 Myr) discless stars (Biller et al. 2013; Brandt et al. 2014) at large orbital radii (> 50 au) suggest that much of the structure is likely to be on small scales. Here the high resolution afforded by ELT class telescopes in conjunction with ALMA will be essential.

\section{Evolutionary processes in protoplanetary discs}

Young low mass stars have been traditionally classified according to the slope of the infrared spectral energy distribution (Lada \& Wilking 1984). It is now widely believed that the resulting classes, which have increasingly steep spectral energy distributions (i.e. less flux at longer wavelengths) represent an evolutionary sequence between objects that are strongly disc/envelope dominated (Class O/I) to those that are - at least approximately - disc-less (Class III). The majority of disc bearing objects discussed so far, and indeed the bulk of the disc bearing population, belong to Class II, a stage where the star is clearly visible in the spectrum in the optical but where there is also clear evidence of emission at longer wavelengths, largely interpreted as stellar radiation reprocessed by disc dust. Despite the uncertainties in disc masses described above, it is very unlikely that the majority of Class II discs are sufficiently massive for the disc's self- gravity to be important compared with that provided by the central star. At the younger (Class 0/I) stage, this is not necessarily the case. Models for the collapse of protostellar cores (Vorobyov et al. 2013) predict that discs should pass through an early self-gravitating phase which is hard to access observationally, both on account of its relative brevity ( $\sim 10^{5}$ years) and the fact that it coincides with a phase when protostellar systems are deeply embedded in dust. It is only extremely recently that ALMA has started to reveal examples of 
objects that are probably in this stage and which exhibit both massive spiral features and, in some cases, the formation of fragments (Tobin et al. 2016; ; Perez et al. 2016). Such observations provide vindication for the large body of theoretical and numerical work which predicts that large amplitude spiral structures (and hence the possibility of disc fragmentation) should be restricted to the outer regions of young protoplanetary discs $(>50 \mathrm{au})$ where the ratio of the cooling time to the dynamical time is short (Gammie 2001; Rafikov 2005, 2009: Clarke 2009): see however Meru \& Bate (2011. 2012); Paardekooper et al. (2011); Rice et al. (2014); Young \& Clarke (2015) for a discussion of the challenging numerical issues involved in modelling protostellar discs during the self-gravitating phase.

In the next decade it can be expected that ALMA will improve our understanding of the earliest phases of disc evolution considerably. Although this phase is brief, it is potentially long enough to allow the formation of planets by gravitational instability in the outer disc (Durisen et al. 2007). Spiral modes in the disc can affect large scale redistribution of material in the disc, and spiral shocks can provide suitable locations for accelerated early grain growth (Rice et al. 2004, Clarke \& Lodato 2009. Booth \& Clarke 2016) and chemical processing (Ilee et al. 2011) . High resolution simulations, with the capacity to resolve the large dynamic range of size scales associated with 'gravito-turbulence' will form an essential theoretical counterpart to new observational discoveries.

Turning now to the more abundant lower mass discs which dominate the population on timescales of Myr, one of the most important evolutionary processes (apart from planet formation itself) is the redistribution/removal of angular momentum from orbiting dust and gas. Any such process drives radial flows, redistributing material in the disc and causing accretion on to the star. Since protoplanetary discs are observed to be accreting at rates which imply a significant fraction of the disc should be lost to the star over a Myr timescale (Hartmann et al. 1998; Manara et al. 2016), it is clear that protoplanetary discs should be considered as accretion discs. What is not clear, however, is the process driving this angular momentum transfer. A front running mechanism for angular momentum redistribution in recent decades has been the magneto-rotational instability (Balbus \& Hawley 1991) a linear instability of weakly magnetised discs under ideal MHD that operates in any disc in which the angular velocity decreases outwards. While this is found to be effective for moderately ionised conditions (Davis et al. 2010; Simon et al. 2012), Gammie et al. (1996) first pointed out that finite resistivity should limit the effective operation of the MRI to regions of suitably high ionisation level and that elsewhere (between $\sim 0.3$ and 10-30au) the disc has an extensive MRI 'dead zone'; there has subsequently been considerable interest in linking the low levels of magnetohydrodynamical turbulence in or at the boundaries of such zones with conditions conducive to planet formation (Regaly et al. 2013: Hu et al. 2016) and to examining how low effective viscosity in such regions affects the accretion and migration history of planetary cores (Matsumura et al. 2007, Hasegawa \& Pudritz 2013).

Recent years have seen the first attempts to characterise the level of magnetohydrodynamical turbulence in discs using spatially resolved observations of molecular line emission (Flaherty et al. 2015, Teague et al. 2015). The analysis involved is 
highly delicate in that the signature of turbulence in line profiles depends on being able to accurately subtract away the line profile that is expected from thermal broadening and Keplerian shear alone. Turbulent levels are found to be low but it is presently unclear whether they contradict the predictions of MRI generated turbulence.

Meanwhile, a recent change in direction has been provoked by simulations which include other non-ideal MHD effects in addition to resistivity. In particular, it has been found that disc regions that were considered to be beyond the traditional MRI dead zone are subject to strong damping of magneto-hydrodynamical turbulence by ambipolar diffusion (Bai \& Stone 2013; Simon et al. 2013, Bai 2013). This suppression of the MRI is so effective that accretion cannot be driven at observable levels unless the disc is threaded by a net vertical field. In this case, however, it is found that instead of small scale magnetoturbulence driving angular momentum transport in the disc plane, angular momentum and mass are instead removed in the form of a large scale magnetohydrodynamical wind. Currently, this conclusion is based on local (shearing box) simulations and a clear goal for the next decade is to establish the reality or otherwise of such flows in global simulations (Zhu \& Stone 2017).

If this picture of large scale magneto-hydrodynamic winds turns out to be correct then it will prompt a paradigm shift concerning our understanding of secular disc evolution and would imply, for example, that discs shrink rather than grow with time and that in principle a significant fraction of disc gas could be ejected rather than being accreted onto the star. Currently efforts to test this scenario observationally are in their infancy and over-lap with efforts to test models for photoevaporation (see below).

The disc bearing (Class II) lifetime of protoplanetary discs is typically in the range of a few Myr (Haisch et al. 2001; Fedele et al. 2010). The subsequent evolutionary stage (Class III) is compatible, from the point of view of the spectral energy distribution, with being essentially disc-less - not only is there no evidence for accretion on to the star, but the lack of near-infrared excess goes hand in hand with undetectably low levels of far infrared and submm emission (Duvert et al 2000, Cieza et al. 2013). For solar mass stars, this places upper limits on the quantity of $\mathrm{mm}$ size dust of less than a few earth masses, which is around an order of magnitude higher than the quantities (see Fig. 2) of such dust detected in the youngest debris discs (Panic et al. 2013). Likewise, CO is not detected in non-accreting T Tauri stars (Hardy et al. 2015), implying that the disappearance of disc dust is correlated with the dispersal of its gas also. It is still unclear what processes drive disc clearing (i.e. effect the change from Class II to Class III status) but one thing that has become obvious is that it cannot be achieved by a simple viscous draining of material on to the star: an extrapolation of observed disc masses and accretion rates in Class II sources would imply that they would then lose their infrared excess over hundreds of Myr and would spend the majority of that period with the colours of optically thin infrared emission. This is contrary to the observational situation (Ercolano et al. 2011; Koepferl et al. 2013), where discs are either largely optically thick in the infrared (although with some transition discs evidencing cleared inner regions 


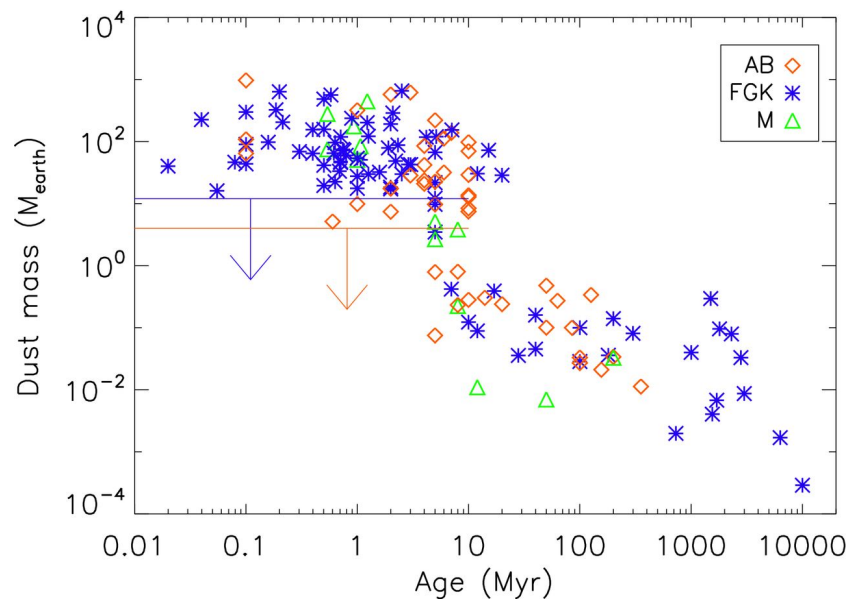

Fig. 2 The evolution of the mass of dust in mm sized grains from the protoplanetary to the debris disc phase, with data colour coded according to the spectral type of the central stars. Protoplanetary and debris discs are well separated in terms of age and dust mass but note the higher upper limits on detectable dust masses in young systems given the typical distances to star forming clouds Panic et al. (2013).

in their spectral energy distributions: see earlier) or else essentially disc-less. Some process, acting on a timescale that is a small fraction of the typical disc lifetime, is responsible for achieving this final dispersal (see Alexander et al. 2014 for a review of possible dispersal mechanisms).

It is currently unclear how transition discs fit into this evolutionary scenario. At the time that they were first identified, first through anomalous spectral energy distributions and then subsequently via targeted imaging, they were believed to represent a minority class, constituting around $10-20 \%$ of all Class II objects. Objects with such cleared inner regions were thus seen as short-lived immediate precursors of disc final clearing. While this picture may still have some merit it has become complicated by the recent insights provided by spectacular images such as the ALMA Science Verification Data on HL Tau (ALMA Partnership 2015) which shows pronounced annular structures in a disc (see Fig. 33 which showed no signature of partial clearing in its spectral energy distribution and which moreover - from its high accretion rate - is thought to be a young system. This unexpected evidence of structure in a disc not previously identified as a transition disc has opened up the possibility that the majority of Class II discs may turn out to be similarly structured, in which case such structure (whether produced by a planet or some other agent) is 

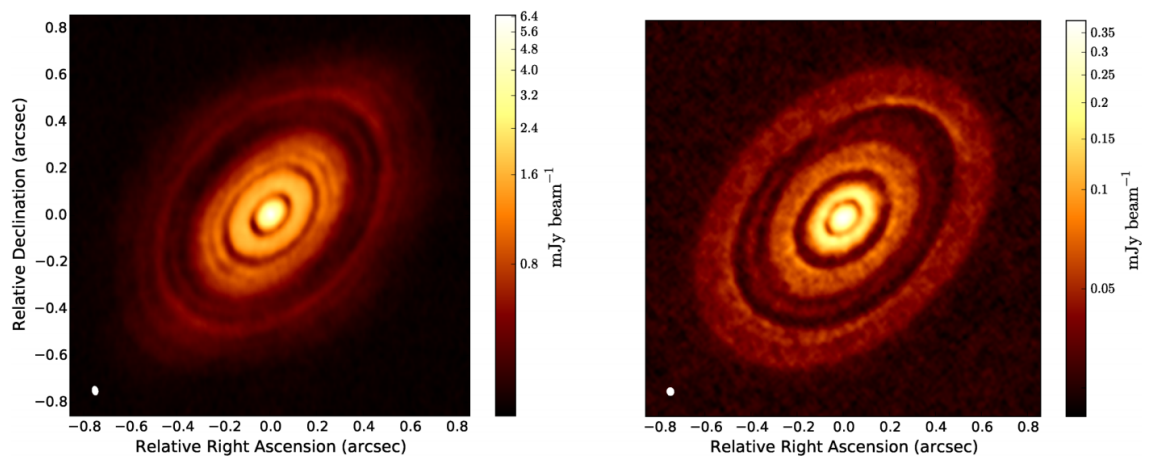

Fig. 3 This famous ALMA 1.3 mm continuum image (ALMA Partnership 2015) of annular dust rings in HL Tau (left) is remarkable in that its spectrum had given no grounds to suspect the presence of such structure. This raises the possibility that many young discs may contain such structure. The panel on the right is a simulation by Dipierro et al. (2015) in which three sub-Jovian mass planets are located at 13,32 and 69au.

not an indication of imminent disc dispersal. Ongoing high resolution imaging programmes with ALMA based on an unbiased sample of Class II discs will do much to clarify the incidence and nature of dusty structures in protoplanetary discs.

Meanwhile there are further ways of exploring the mechanism for disc dispersal through examining the evidence for disc winds. Such winds may represent the MHD winds described above or else photooevaporative winds driven by ultraviolet/X-ray radiation, either from the central star or the star forming environment. The theory of photoevaporation is well developed compared with that of MHD winds (see Clarke et al. 2001; Alexander et al. 2006, Owen et al. 2010, 2012; Gorti et al. 2015, in the case of evaporation by the host star and Johnstone et al. 1998, Adams et al. 2004: Facchini et al. 2016 for photoevaporation driven by neighbouring higher mass stars). Photoevaporative winds are predicted to be a significant sink of mass at radii beyond a few au and to be important agents of dispersing the last remnants of disc gas at late evolutionary stages of protostellar disc evolution. The narrow components of a number of optical and near-infrared lines in protostellar discs (such as NeII and OI) can be explained by photoevaporation models (Alexander 2008, Ercolano \& Owen 2011) . To date there has been no similar exploration of the observability of MHD winds, in part on account of the lack of self-consistent global models and in part because of the observational difficulty of disentangling the signatures of such winds (which can launch from the inner disc at hundreds of $\mathrm{km} \mathrm{s}^{-1}$ ) from that of similarly high velocity outflows associated with jets.

Mapping at $\mathrm{cm}$ wavelengths using the VLA or e-Merlin can potentially provide observational constraints on the rate of mass loss in ionised flows since imaging in the free-free continuum can be used to map the distribution of extended ionised gas around protostellar discs. Currently the resolution attainable (10s of au) offers the possibility of distinguishing between photoevaporative winds driven by EUV radiation and the denser conditions produced in the more vigorous $\mathrm{X}$-ray driven 
winds. Such observations will also be confronted with the predictions of global MHD wind models as these become available.

\section{Debris discs}

Debris discs are the circumstellar discs found around main sequence stars. They are made up of asteroids, comets, dust and gas, all of which may be interspersed within a planetary system. Planetesimals in these systems collide and are ground down to dust that is readily detectable through the infrared (IR) excess it creates. Thousands of such debris discs are known and for around a hundred, we have been able to make a resolved image of these discs. Many of these planetesimal belts are cold $(T \lesssim 100 \mathrm{~K})$ and observed in the far-infrared (as such, they may be considered analogues to the Kuiper belt in our Solar System). However, dust very close to its host star ( $T \gtrsim 300 \mathrm{~K}$, analogous to the Solar System's zodiacal cloud) is also observed around a significant fraction of stars. In addition, gas is detected in a growing number of debris discs. Moreover, dust and gas are also observed around the oldest stars that had time to transform into white dwarfs.

Far-IR emission from debris disc dust is found around stars of all spectral type. Detection rates are around 20\% for A-K spectral types, with some evidence for a fall-off in rate toward later spectral types (Eiroa et al. 2013, Thureau et al. 2014). The debris discs that are detected are much more massive than our Solar System's Kuiper belt. There are few dust detections for M-stars, but this does not mean that M stars do not have discs, since the low luminosity of such stars reduces the detectability of any emission in the current surveys. The improved sensitivity of future far-IR missions (like SPICA) offers the potential to discover more discs around these latetype stars. The paradigm to explain the observed cold dust emission is that the dust is produced from a reservoir of big planetesimals that slowly depletes and grinds down to dust in a process known as a collisional cascade. This is supported by the (on average) lower infrared emission from older stars. The size of the biggest bodies composing the belt is not well-known but should be large enough (at least a few km in diameter) for the belt to collisionally survive for billions of years as observed (i.e. $\gtrsim 10 \mathrm{~km}$, Löhne et al. 2008). These discs can be considered as the left-overs of the planet formation process, most of which occurred in the protoplanetary disc phase described in the previous section. As such debris discs provide information on the outcome of the planetesimal and planet formation processes that went on at earlier epochs. 


\section{Birth of debris discs}

There are still many unknowns as to the origin of debris discs and the different steps from the protoplanetary disc phase (see previous section) leading to their creation (see Wyatt et al.2015, for more detail). For example, it is not yet clear if the planetesimals that replenish the dust seen in debris discs are already present early on in the protoplanetary disc phase (e.g. in the rings observed in HL Tau or TW Hydrae, see Fig. 3). Our ignorance is driven by the difficulty of detecting the planetesimals, but also by a lack of understanding of how to overcome the bouncing barrier (Blum \& Münch 1993) and radial drift (Weidenschilling 1977) that otherwise prevent sub$\mu \mathrm{m}$ sized dust in protoplanetary discs growing beyond $\mathrm{cm}$ in size. One solution is that planetesimals form through gravitational instabilities in dense regions of the protoplanetary disc, perhaps at locations where dust density has been enhanced by the streaming instability (an instability in which dust grains concentrate into clumps owing to gas drag leading to their gravitational collapse, Chiang \& Youdin 2010, Johansen et al. 2014). This may mean that planetesimals form at favoured locations in the protoplanetary disc (e.g., near snow-lines, Schoonenberg \& Ormel 2017), or just outside the gaps carved by planets or in spirals of gravitationally unstable discs (see Fig. 1). However, since the streaming instability is enhanced when the gas is depleted relative to the dust (Carrera et al. 2015), it is possible that planetesimals are preferentially formed late on, while the disc is in the process of having its gas dispersed by photoevaporation (Carrera et al. 2017).

These different possibilities make different predictions for the radial location and radial width of the region in which planetesimals would be expected to form which can be compared with the observed properties of debris discs. Studies of nearby debris discs around main sequence stars with a range of ages tell us that planetesimal belts can be present at up to $\sim 200 \mathrm{au}$, but the known debris belts are more commonly closer in at $\sim 40 \mathrm{au}$. They are also often in belts that are radially narrow $d r / r=0.1$ (e.g., HR 4796, HD 181327, Fomalhaut; see Fig. 4), although there are examples of broad belts too $d r / r>1$ (e.g. $\beta$ Pic, see Fig. 5), and systems with belts at multiple radii (e.g. Ricci et al. 2015). These observations already provide distributions that can be used to constrain models of planetesimal formation, though such comparisons are only just beginning to be made. However, high resolution imaging of a larger sample of debris discs with ALMA, and later with the coming radio array SKA, will provide better constraints on the location and width of the planetesimal belts in these systems (which is usually inferred indirectly from the emission spectrum or shorter wavelength data).

Studies of young associations (e.g., the TW Hydra association, or the $\beta$ Pic moving group) allow to probe the properties of debris discs straight after their formation. These show a diversity of disc radii and widths that is not significantly different to that of the discs of older main sequence stars. Thus, there is no evidence for an increase in disc radius with age (Najita \& Williams 2005) as expected in models in which a debris belt only becomes sufficiently luminous to be detectable once sufficient time has elapsed for planetesimals to grow into Pluto-sized objects (which takes longer further from the star, Kenyon \& Bromley 2008). However, there are 
relatively few debris discs known at this early epoch, and further studies of debris discs close to the transition are needed to assess if there is any evolution other than the decrease in brightness expected from collisional grinding. With the help of ALMA and future potential missions such as SPICA, or a 10m-aperture far-IR surveyor (such as the one proposed by NASA called the Origins Survey Telescope) it will be possible to identify and characterise debris disc dust levels in nearby star forming regions.

The evolution of the debris in the transition phase is poorly constrained at present, yet the dynamics of this transition can result in observable (i.e., testable) phenomena. For example, the dispersal of the protoplanetary disc may sweep the remaining $\mathrm{mm}-\mathrm{cm}$ sized dust into belts (Alexander \& Armitage 2007) that, assuming this mass does not coalesce into planetesimals, would be both luminous and short-lived. Sweeping can also occur through interaction with planets that formed closer in, since these may undergo migration shortly after formation (Wyatt|2003, Capobianco et al. 2011) or be scattered into an outer planetesimal belt. Bright rings of $\mu \mathrm{m}$-sized dust can also be created without in situ planetesimals through the action of gas drag on such small dust (Takeuchi \& Artymowicz 2001). This simply requires an inner planetesimal belt and a substantial gas disc, and may possibly explain the two narrow rings seen in scattered light at 100s of au in HD 141569 despite mm-sized grains not being detected at these locations (White et al.2016). Again, further observations of systems in the late phases of protoplanetary disc evolution, or in the early stages of debris disc formation, will help to understand this transition.
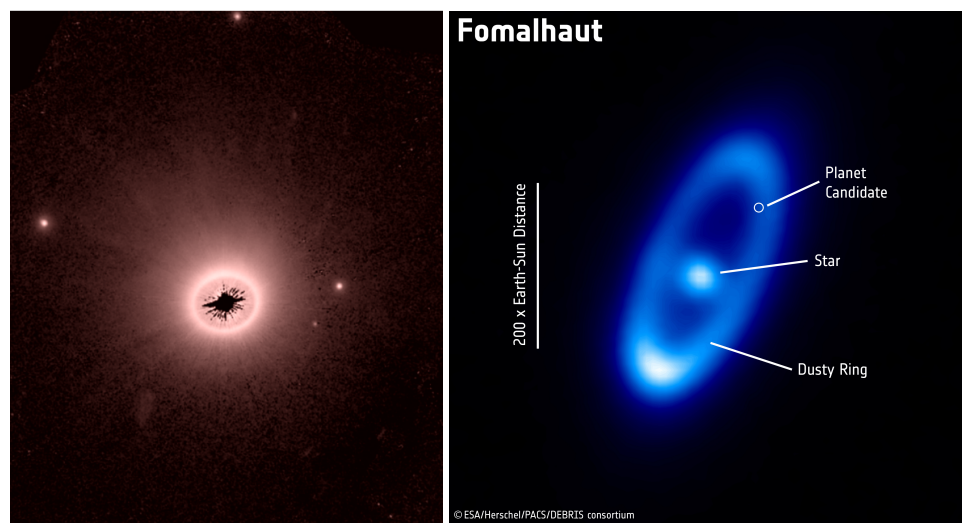

Fig. 4 Two ring-like debris discs: left) HD 181327 observed with HST (Schneider et al. 2014); right) Fomalhaut observed with Herschel (Acke et al.2012).

Another property of the known debris discs is that their planetesimals must collide at high enough velocities to create significant quantities of dust. Since collision velocities are expected to be low in a protoplanetary disc due to damping by gas drag, there must be some process that stirs the planetesimal belt. Possibilities include that the planetesimals inherit a large velocity dispersion from their formation 
process (e.g. Walmswell et al.|2013), or from some other (as yet undefined) aspect of protoplanetary disc evolution, or dispersal causes them to end up with a large velocity dispersion as soon as the protoplanetary disc has dispersed. Stirring could also occur after protoplanetary disc dispersal; e.g. the planetesimals could be born with a low velocity dispersion resulting in their growth into Pluto-sized objects that stir the disc (i.e., self-stirring, Kenyon \& Bromley 2001, Kennedy \& Wyatt 2010), or an interior planetary system could stir the disc (Mustill \& Wyatt 2009). Population studies of debris discs are inconclusive as to the origin of the stirring, but detailed investigations of individual systems allow constraints to be set within the context of the different scenarios on, say, the mass and orbit of the perturbing planet, or the surface density and initial planetesimal sizes for a self-stirred disc.

Such detailed studies of individual discs can also provide information on the level of stirring. For some systems the vertical height of edge-on discs suggests a low level of stirring ( $<5 \%$, e.g. AU Mic, Krist et al. 2005), while a sharp outer edge in others suggests likewise (Thébault \& Wu 2008). Herschel also discovered a population of cold debris discs (Eiroa et al. 2013) that are best explained as unstirred debris belts of planetesimals that are 10s of metres in size that evolve very slowly but producing low quantities of dust (Krivov et al. 2013). However, the possibility that the emission from these cold discs arises from background galaxies still needs to be unambiguously ruled out (Gáspár \& Rieke 2014), perhaps by confirming that the emission is co-moving with the stars in question. Overall, high resolution studies of larger numbers of discs are required to determine the stirring level and its dependence on other properties of the system.

\section{Links between debris and planets}

There are currently $\sim 40$ systems known to host both a debris disc and a planet (Marshall et al. 2014, Moro-Martín et al.|2015). Since both debris discs and planets are thought to form in protoplanetary discs, it might be expected that the properties of these two components should be correlated somehow, for example because the protoplanetary disc properties that are favourable for forming planets might also be conducive for the formation of planetesimals. However, early studies found no such correlation (e.g. Bryden et al.2009), which was attributed to the fact that the known debris discs are typically located at a few 10's of au, while planets are typically much closer in. More recently tentative evidence has been found for a correlation between the presence of low-mass planets detected in radial velocity surveys and debris (Wyatt et al.|2012; Marshall et al.|2014), and a possible anti-correlation with giant planets which could more easily scatter any debris that would remain (Raymond et al. 2012). It is also notable that most of the systems with planets that have been directly imaged at $\gg 5$ au also have debris discs, the most famous being $\beta$ Pic (see Fig. 5) with a $\sim 7 \mathrm{M}_{\text {Jup }}$ planet at $\sim 9$ au (Currie et al. 2013), HR 8799 with 4 detected planets sandwiched between inner and outer debris belts (Marois et al.|2008. 
2010; Matthews et al.|2014; Booth et al. 2016), and Fomalhaut with a narrow debris ring and an eccentric planet (Kalas et al.2013).

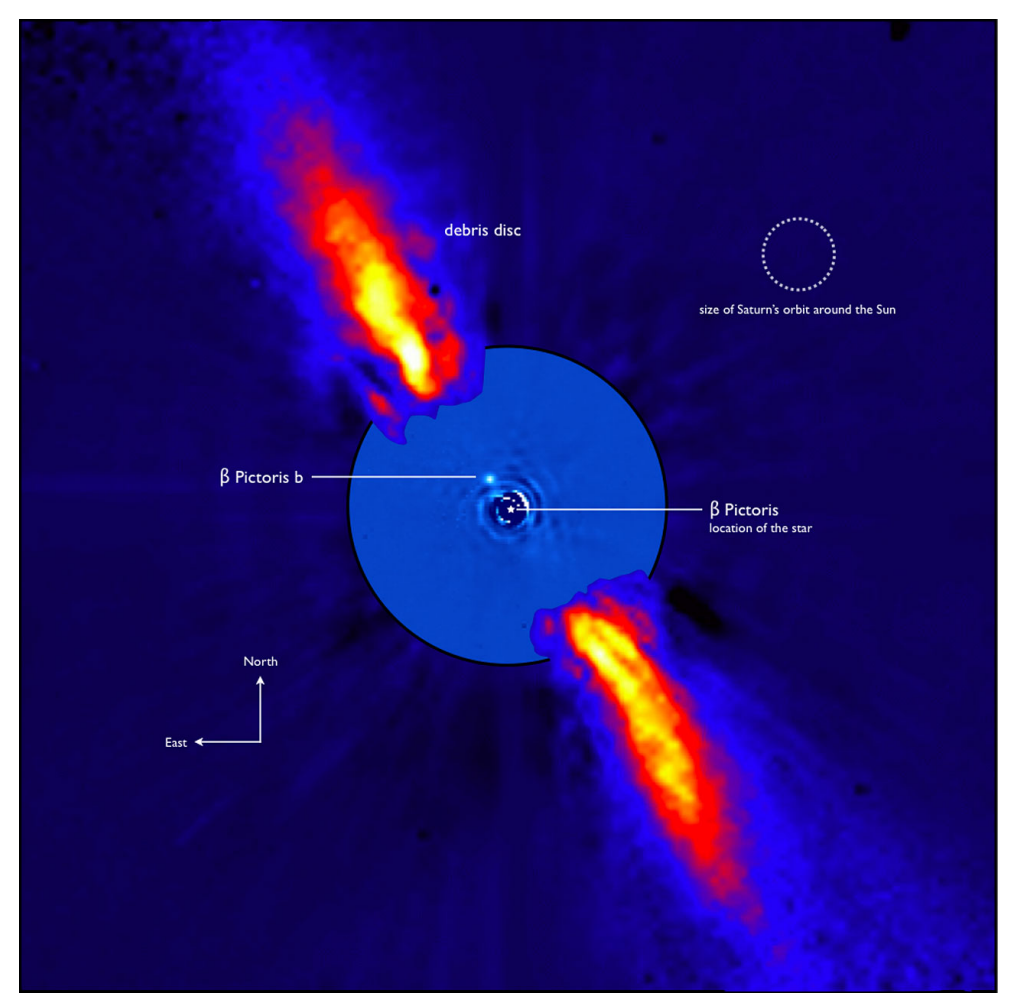

Fig. $5 \beta$ Pic's debris disc and planet. This is a composite image in the near-infrared for which the ADONIS instrument on ESO's $3.6 \mathrm{~m}$ telescope was used to observe the outer part of the disc and the inner part is seen using the NACO instrument on the VLT. The detected planet $\beta$ Pic b is at a projected distance of $\sim 9 \mathrm{au}$ and has a mass about 7 times that of Jupiter. ESO/A.-M. Lagrange et al.

Our understanding of the links between debris and planets could be extended on two fronts, by using statistical methods to identify correlations between the properties of a system's debris disc and its planetary system, and by using resolved images of debris discs to characterise the way in which planets interact with debris and to identify planets that could not have been detected otherwise (and to learn about their formation history). The current statistical studies mentioned above are limited by the small number of detections, both of debris and of planets. This will improve significantly with future far-IR missions that can detect debris around more distant stars (and so increase the number of known debris discs) as well as detect debris discs down to fainter levels approaching that of the Solar System's Kuiper belt. A variety of exoplanet detection techniques will also increase the number of known exoplanets. For example, Gaia is expected to discover at least 20,000 planets (more 
massive than Jupiter with periods out to several au) within 500pc (Perryman et al. 2014), meaning that 1000's should be discovered in systems with a bright debris disc, the closest of which will be known from Herschel surveys (out to 20-50 pc), a distance limit which can be extended in future far-IR surveys. Upcoming transit missions will also be able to detect small Earth-like planets in edge-on systems: TESS is expected to detect $\sim 2000$ planets (among which 500 will have a radius smaller than twice the Earth, Sullivan et al. 2015), and PLATO is expected to detect 100's of earth-like planets and 1000's greater than Neptune (Rauer et al.|2014). Some of these systems will have constraints on their debris disc from far-IR surveys, but these are also good candidates to search for correlations with hot dust co-located with the planets (see next section).

If there are planets in a system hosting a debris disc then it is inevitable that the planets' gravitational perturbations will impose structure on the debris disc. It is known that planet-disc interactions can create detectable asymmetries: clumps (Wyatt|2003), offsets (Wyatt et al. 1999), warps (Augereau et al.|2001), gaps (Shannon et al. 2016), spirals (Wyatt 2005). Our understanding of the variety of features that planets can produce is also growing; e.g., planets can also create scattered-disc analogues (similar to the one in our Solar System mostly populated by icy minor planets at $>30 \mathrm{au}$ ) and mini-Oort clouds that are potential outcomes of planetary systems (see Wyatt et al. 2017). Thus observations of such features in debris disc images can provide evidence for planets that would otherwise be undetectable. For instance, in $\beta$ Pic (see Fig. 5), the detected planet was first hypothesised because of the observation of a warp in the $\beta$ Pic dust disc (Mouillet et al. 1997). Another example is the growing number of detections of eccentric discs, which is thought to be due to the presence of eccentric planets secularly forcing the disc to become eccentric over long timescales (Wyatt et al. 1999), Lee \& Chiang|2016).

In the coming years the number of features observed in debris discs will grow as new instrumentation becomes available. ALMA is already providing images of structures in the parent planetesimal belts of some systems. The small inner working angles, high-resolution and contrast of new instruments such as SPHERE or GPI are also providing scattered light images that reveal new structures not foreseen by models with no easy interpretation (e.g. AU Mic, Boccaletti et al. 2015). Scattered light imaging capability will continue to improve with JWST, WFIRST and the ELT, and thermal imaging capabilities will improve with ALMA, JWST and SKA. A multi-wavelength approach is particularly crucial to test models for the origin of a given structure, since the interpretation of a given feature is often degenerate (e.g. Wyatt 2006). For example, a dust and gas clump like that seen around $\beta$ Pic (Dent et al. 2014: Matrà et al. 2017a) can arise from resonances with a planet or from a single massive collision at 10's of au (Jackson et al.2014; Kral et al.2015), although in this case the breadth of the gas clump rules out a giant impact origin (Matrà et al. 2017a).

Unambiguously identifying debris disc structures with known planets is important to test and further refine our understanding of planet-disc interactions (e.g. Thebault et al. 2012) and the processes of their formation and evolution. This gives a much better handle on the origin of these structures (linked to planets or not) and 
allows us to better constrain debris disc models and refine some of the physics used in these models. While this is challenging because the planets are often hard to find with other methods, the brightest planets can be detected in direct imaging, the capabilities for which are improving with the same instrumentation used to image the disc (i.e., JWST, WFIRST, ELT). Even if detections of low mass planets (i.e., Neptunes) in the outer regions (i.e., $>5 \mathrm{au}$ ) of specific systems may remain challenging, our understanding of the frequency of such planets will be transformed by the microlensing surveys of EUCLID and WFIRST, and this will significantly inform our interpretation of debris disc structures.

\section{Debris in the middle of planetary systems}

While most debris discs are made up of a cold belt at 10's of au, we know of the existence of many two-temperature debris discs that are mainly probing systems with multiple belts such as the Kuiper belt and the Asteroid belt in our Solar System (Kennedy \& Wyatt 2014). Dust within a few au of its host star is also observed around a large fraction of systems irrespective of the existence of a cold outer belt. When this dust is warmer than around $300 \mathrm{~K}$ it is referred to as an "exozodi" in reference to the zodiacal dust in our Solar System that surrounds the innermost planets and goes all the way to a few solar radii. We distinguish hot dust (up to $\sim 2000 \mathrm{~K}$, very close to the host star) and warm dust $(\sim 300 \mathrm{~K}$, in the habitable zone of the system) from an observational perspective as the former is observed in the near-IR and the latter in the mid-IR. Current near-infrared interferometry studies have detected hot dust around $>10 \%$ of stars (Ertel et al. 2014), with surprisingly little dependence on the properties of the host star or its outer debris belt. Mid-infrared photometry has shown that bright warm dust (brighter than around $10 \%$ of the stellar photospheric level at $12 \mu \mathrm{m}$ ) is relatively rare around old nearby stars but more common around young stars (Kennedy \& Wyatt 2014). However, mid-infrared interferometric techniques show that lower levels of dust (at the $0.1 \%$ above photospheric level with the Keck Interferometer Nuller) may correlate with the presence of an outer debris belt (Mennesson et al.|2014).

The origin of exozodi dust is uncertain at present (see the review by Kral et al. $2017 \mathrm{~b}$ for more details). The high luminosity and temperature of the hot dust defies easy explanation, because its collisional depletion at its inferred proximity to the star prevents its accumulation. One of the proposed explanations involves magnetic fields trapping nano-grains (Rieke et al. 2016), underlining that the physics in these highly collisional and hot systems may vary from typical colder belts. Warm dust that can be at larger distance from the star is easier to explain. For young stars $(<100 \mathrm{Myr})$ with dust within a few au, the favoured explanation is in a massive collision, which like the Moon forming collision with Earth is expected in the late stages of planetary formation (e.g. Kenyon \& Bromley 2006; Raymond et al. 2009, Jackson \& Wyatt 2012). Thus warm dust detections provide a way of probing ongoing planetary formation (e.g. Lisse et al. 2009), an interpretation which is sup- 
ported by photometric variations (on $<1 \mathrm{yr}$ timescale) in the infrared (see Fig. 6 , Meng et al. 2014, 2015). However, for older stars ( $>100 \mathrm{Myr}$ ), such giant impacts are expected to be rare, and collisional depletion precludes detectable levels of warm dust having its origin in an in situ asteroid belt (Wyatt et al.|2007a). Instead the observed warm dust could be supplied from an outer planetesimal belt, either through Poynting-Robertson drag that transports the dust inward (van Lieshout et al.|2014; Kennedy \& Piette 2015), or through the scattering in of planetesimals by interaction with planets resulting in exocomet activity (Bonsor et al. 2014, Faramaz et al. 2016). These possibilities are supported by the aforementioned correlation of bright mid-infrared excesses with outer belts. For warm dust that is sufficiently far from the star, these can be explained as extrasolar analogues to the asteroid belt (e.g. Geiler \& Krivov 2017). A new generation of models (e.g., Kral et al. 2013), along with the new observations of this phenomenon (discussed below), will eventually unravel the origin of these excesses.

Regardless of its origin, this dust is a valuable probe of the innermost regions of planetary systems where habitable planets are located. However, it is also a potential hindrance to the direct detection of Earth-like planets in the habitable zone; e.g., even a small amount of dust (10-20 times brighter than our faint zodiacal cloud) could severely hamper detections (Defrère et al. 2010). As such, there is currently much effort in trying to characterise dust in the habitable zone of nearby stars down to much lower levels; e.g., the NASA HOSTS survey is using the LBTI nulling interferometer to search the nearest stars for mid-infrared excesses down to $0.05 \%$ above the photospheric level (Defrère et al. 2016), with the aim of identifying exozodi-free targets that are most suitable for future searches for Earth-like planets. While LBTI provides limited information on the spatial distribution of any exozodi that it finds, it may provide the first hints of the clumpy structures expected as dust interacts with an inner planetary system (Shannon et al.|2015). JWST also has the potential to detect the asymmetric dust distribution expected to persist for Myr from giant impact debris (Kral et al. 2015), and to search for any photometric variability. Future direct imaging missions such as WFIRST will also provide the very small inner working angle (0.1"), high resolution and constrast needed to resolve these Solar System analogues, a capability which will be further improved upon by ELT. Second generation VLTI instruments such as GRAVITY and MATISSE (together with the current PIONIER) will lead to multi-wavelength measurements over a large range of dust temperatures (see Fig. 7) that may reveal a connection between the hot and warm dust and will explore dust properties through the potential detection of spectral features (e.g. the 3 and $10 \mu \mathrm{m}$ silicate features, Ertel et al.|2012). VLTI in the southern hemisphere complements the LBTI in the north but is not as sensitive. A new concept instrument on the VLTI using nulling interferometry (to get rid of the stellar contribution) may be built in the future to improve the high constrast capabilities needed to detect these excesses. In the distant future, improved constraints would be possible with space-based infrared interferometry (e.g. Fridlund 2004; Labeyrie 2016).

Other novel ways are also being proposed to probe hot and warm dust. For example, small dust clumps embedded in exozodis whose IR-excesses cannot be de- 
tected by current instruments can mimic an Earth-like astrometric signal (Kral et al. 2016a), though can be distinguished from a planetary signal by multi-wavelength observations. Variability on timescales of at least as short as one year has also been found for some hot exozodis (Ertel et al.2016); characterising this variability is the first step to understanding its origin. Polarisation measurements may also be used to find new exozodis (e.g. Marshall et al. 2016). The detection of falling evaporating bodies (through high-velocity gas absorption lines) may also be a good tracer for the presence of hot dust (Beust \& Morbidelli 2000; Welsh \& Montgomery 2015, Eiroa et al. 2016). Dips and dimming in the light-curves of nearby stars, such as that already detected by Kepler (Boyajian et al. 2016), which may be detected more commonly by PLATO, may have its origin in transits of exocomets in front of the star, thus providing another way of probing planetesimal and dust in the inner regions of planetary systems.

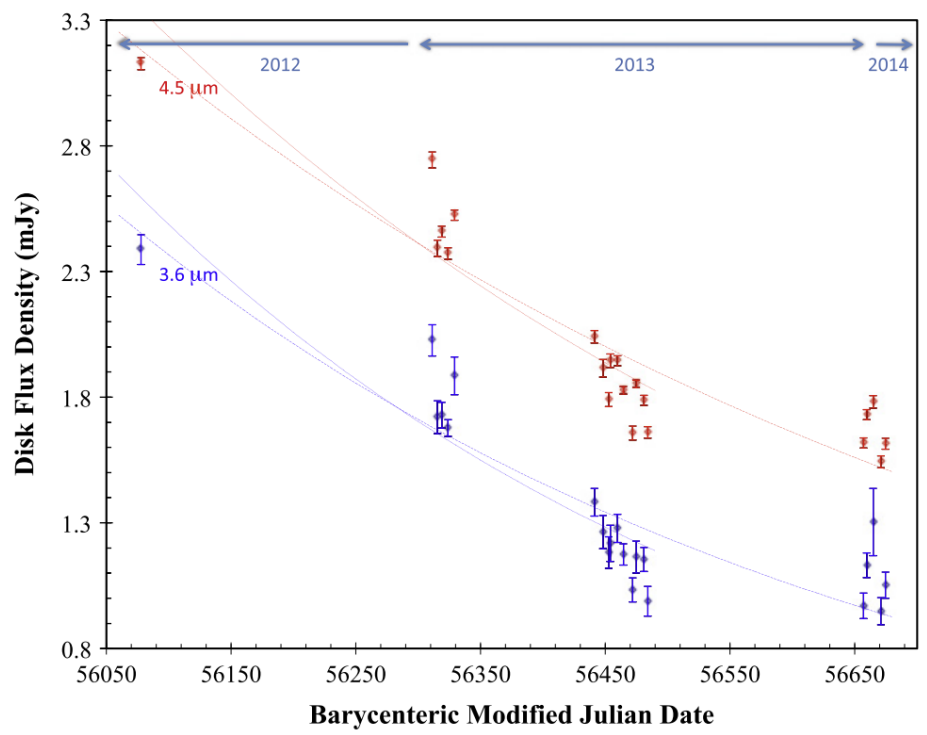

Fig. 6 Flux decrease at 4.5 (red) and $3.6 \mu \mathrm{m}$ (blue) in the P1121 debris disc over more than 2 years. The solid lines are fit to the data assuming an exponential decay (Meng et al. 2015).

\section{Gas in debris discs}

Gas has been discovered around a growing number of main sequence stars, old enough for their protoplanetary gas discs to have dispersed via accretion, photoevaporation or MHD winds (see previous section on evolutionary processes in protoplanetary discs). Molecular CO has been detected in the submm in more than 10 


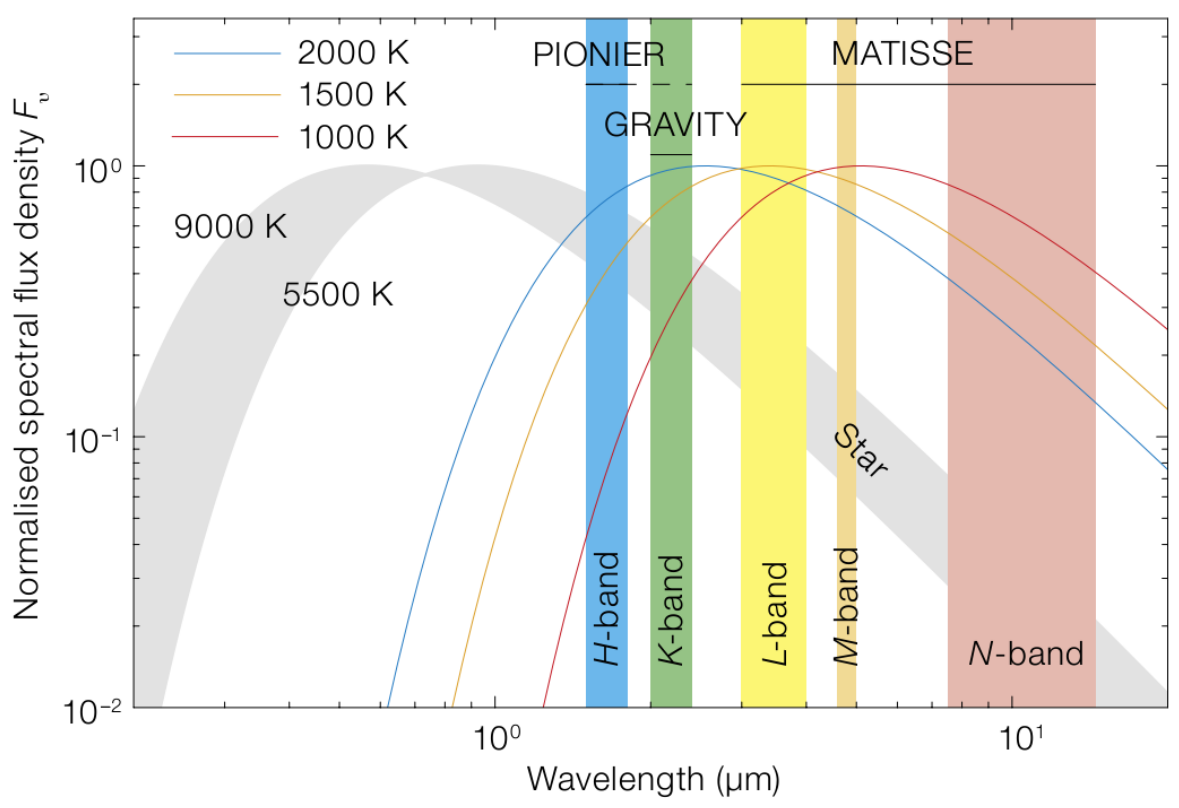

Fig. 7 Wavelength coverage of the second-generation VLTI instruments (and PIONIER) compared to the wavelength range in which blackbody dust emission from hot exozodiacal dust peaks (Ertel et al.2015).

debris discs and is often co-located with its planetesimal belt (e.g. Zuckerman et al. 1995; Zuckerman \& Song 2012; Moór et al. 2015b; Greaves et al. 2016; Matrà et al. 2017a). Atomic carbon and oxygen (e.g. Roberge et al.|2014), as well as metals (Nilsson et al. 2012; Hales et al.2017), have also been detected in a few systems and seem more extended than CO. Atoms have been detected both with the space-based telescopes HST and FUSE through UV absorption lines (Roberge et al. 2000, 2006), with Herschel through far-infrared emission lines (Cataldi et al. 2014: Brandeker et al. 2016), and in the submm (Higuchi et al. 2017). Most of these observations can be explained by a model in which $\mathrm{CO}$ is produced from volatile-rich solid bodies located in the debris belts (as first proposed by Moór et al. 2011; Zuckerman \& Song 2012), which then photodissociates quickly into $\mathrm{C}$ and $\mathrm{O}$ that evolve by viscous spreading (Xie et al. 2013; Kral et al. 2016b, 2017a). The implication is that for most (but not all) systems there is no requirement for primordial gas to be retained from the protoplanetary disc phase, and any debris disc with icy planetesimals will create second generation $\mathrm{CO}, \mathrm{C}$ and $\mathrm{O}$ gas at some level. As such, measurement of these gaseous components provides a way to infer the composition of the exocomets from which the gas was created (Matrà et al. 2015, Kral et al. 2016b, Marino et al. 2016; Matrà et al.|2017b).

To understand the exact dynamics of this gas, its origin, and what we learn from its observations for the planetary system as a whole, a bigger sample of gas detec- 
tions is required. In the immediate future, ALMA is predicted to provide at least 15 new CO detections and 30 new CI detections (see Fig. 8). CI observations are particularly promising, since in addition to being more readily detected than other species, this component spreads viscously all the way to the star potentially enabling resolved imaging of gas disc structures created by close in giant planets. The next generation of far-IR missions, such as SPICA, are predicted to enable detection of at least 25 new systems in CII and $\sim 15$ in OI (Kral et al.|2017a), while a 10m far-IR space telescope could result in $>100 \mathrm{CII}$ gas disc detections, $>50$ systems with OI detected, some of which potentially resolved. The expected level of OI gas depends strongly on the amount of water released together with $\mathrm{CO}$ from the planetesimals, since this provides extra oxygen in the gas disc from the photodissociation of $\mathrm{H}_{2} \mathrm{O}$. This illustrates how gas observations can provide an estimate of the $\mathrm{CO} / \mathrm{H}_{2} \mathrm{O}$ ratio on exocomets that could be compared to Solar System values (i.e., leading to the taxonomy of exocomets). The secondary gas generation process is also expected to create hydrogen (Kral et al. 2016b), accretion of which onto the star has been confirmed observationally for $\beta$ Pic (Wilson et al. 2016), with similar detections possible in other systems. The combination of $\mathrm{CO}$ and $\mathrm{CI}$ or CII detections will lead to a better understanding of the gas dynamics in these discs, by providing estimates of the disc viscosity and ionisation fraction with which to test theories for how angular momentum is transported in the discs (e.g. Kral \& Latter 2016).

$\beta$ Pictoris is so far the only system for which we have spatially resolved images (using VLT/UVES) of the metals (such as NaI, FeI and CaII, Brandeker et al.|2004), which are shown to extend inwards to at least $\sim 10 \mathrm{au}$. The high angular and spectral resolution of UVES should be used on other targets in the future to detect more systems with gas. This technique was first used in Olofsson et al. (2001) and the model by Zagorovsky et al. (2010) can be used to make predictions of the different emission line fluxes expected. These new gaseous systems could then be followedup with more detailed UVES/CRIRES or ALMA observations. This will enhance our understanding of the origin of metals and how they dynamically evolve in these gas discs.

Other novel ways of observing gas around main sequence stars could be through detecting rovibrational CO lines with the JWST as was already done from the ground for $\beta$ Pic (Troutman et al. 2011). HI or OH may also be detectable with future radio telescopes such as SKA (Aharonian et al. 2013) or the next generation VLA (Carilli et al. 2015). For systems that are edge-on, using UV absorption lines (e.g. Roberge et al. 2000) could also enable us to detect new systems with gas. Systems showing the presence of falling evaporating bodies (similar to comets on sun-grazing orbits) through high-velocity gas absorption lines (e.g. Kiefer et al. 2014b a: Montgomery \& Welsh 2012) should be edge-on and may be promising targets for future UV absorption line surveys targetting circumstellar gas (which would benefit from a new generation UV surveyor).

The CO mass for some systems seems likely high enough that it cannot be explained with a secondary gas scenario; the gas in these systems may be of primordial origin and may still contain $\mathrm{H}_{2}$ that (together with $\mathrm{CO}$ ) shield $\mathrm{CO}$ from being photodissociated (e.g. in HD 21997, Kóspál et al. 2013). In the future it will be 


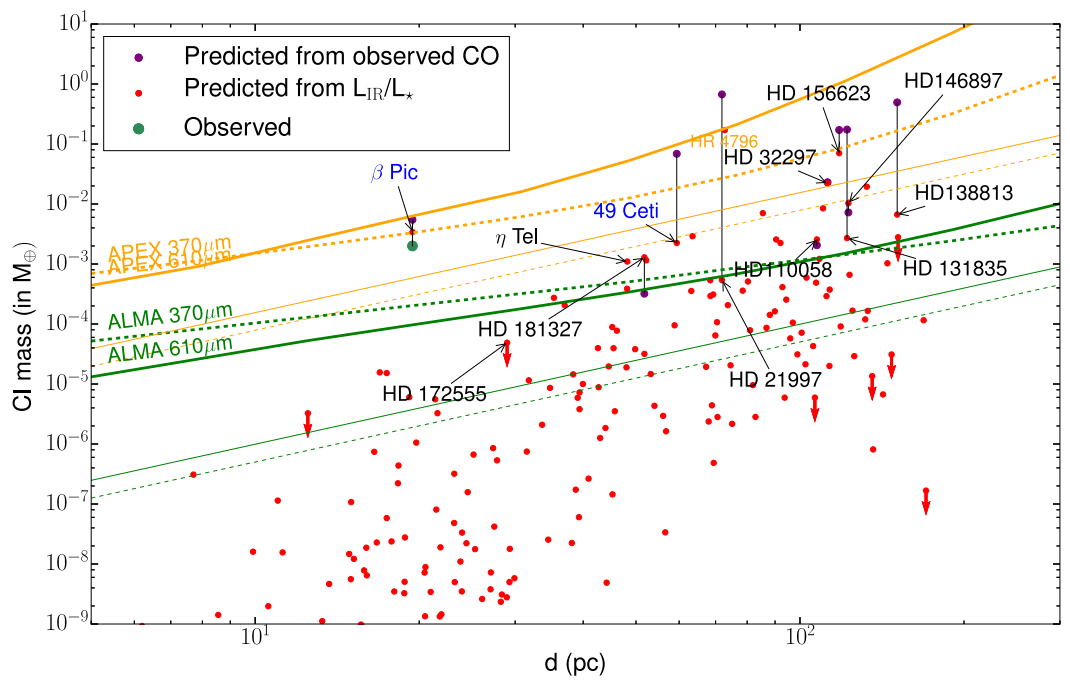

Fig. 8 CI mass (in $\mathrm{M}_{\oplus}$ ) as a function of distance to Earth $(d)$ from the secondary gas model of Kral et al. (2017a). Planetary systems with gas detections are labelled with their names. If CI is already detected the label is in blue (black otherwise). The CI mass for $\beta$ Pic derived from Kral et al. (2016b) is shown as a green point. The red points are predictions from the model. The red downward arrows show systems with grains that are warmer than $140 \mathrm{~K}$, which may not be able to keep CO trapped on solid bodies. The purple points show predictions from the model when the observed CO mass is used rather than the CO mass predicted from $L_{\mathrm{IR}} / L_{\star}$. Detection limits at $5 \sigma$ in one hour are shown for APEX (in orange) and ALMA (in green) at 370 (dashed) and $610 \mu \mathrm{m}$ (solid). The thin lines are for LTE calculations and thick lines for more realistic NLTE calculations (see details in Kral et al.|2017a).

important to identify these primordial gas systems. Understanding why these systems evolved differently from others of similar age will provide vital clues on the transition from the protoplanetary to the debris disc phase (see previous section) and on the origin of debris discs themselves (see previous subsection on the birth of debris discs). A promising way to identify systems with secondary origin (rather than primordial) is to measure an optically thin $\mathrm{CO}$ or CI line ratio (with ALMA for instance) to check that the gas is out of LTE (Matrà et al. 2015), i.e. to show that the disc does not contain the abundant $\mathrm{H}_{2}$ colliders expected in a protoplanetary disc (Matrà et al.2017a).

\section{White dwarf polluted discs}

Practically all known planet host stars (including our Sun) will evolve into white dwarfs (WD). Spectra show that the atmospheres of $\sim 30 \%$ of WD are polluted by 
metals which should not be present due to the short sinking times (Koester et al. 2014). The best explanation for this pollution is that it comes from the tidal disruption of planetesimals originally residing in a cold outer belt, which may have been dynamically perturbed by surviving planets (Farihi 2016), Veras 2016). This is supported by the fact that roughly $2 \%$ of WDs also show an IR-excess consistent with circumstellar rings of dust orbiting close to the tidal disruption radius for these stars (Bonsor et al. 2017). Moreover for one system (WD 1145+017), regular occultations of the star suggest the presence of planetesimals close to the tidal disruption (Roche) limit (Vanderburg et al.2015). In addition to the absorption lines that are characteristic of WD pollution, gas emission lines are observed for $\sim 10 \mathrm{WDs}$, inferred to originate in gas that is both very close to the WD and varies with time (see Fig. 99.

Despite a growing body of observational evidence, our understanding of the processes leading to the accretion of planetesimals is poorly understood. Nevertheless, observations of WD pollution provide key constraints on the mineralogy of rocky exo-planetary material. This is because photospheric metal abundances should trace the bulk composition of accreted planetesimals (Zuckerman et al. 2007). For example, by providing key ratios such $\mathrm{Mg} / \mathrm{Si}$ for the accreted planetesimals, WD pollution measurements provide strong evidence for differentiation in planetary building blocks (Jura \& Young 2014), which supports models for planet formation (e.g. Bonsor et al. 2015: Carter et al. 2015). Such measurements also provide a potential opportunity to search for signatures of geological processes (e.g. plate tectonics) in exo-planetary systems, and complement the on-going programs to detect terrestrial planets over the next decade (see subsection on the links between debris and planets).

For now, $\sim 15$ WDs have at least five detected pollutant elements in their atmospheres (Jura \& Young 2014). This number is set to triple in the next decade from current and on-going observational programs on HST and VLT (X-shooter), but would benefit greatly from a new FUV mission. Ground-based observations can reveal $\mathrm{Ca}$ (as well as $\mathrm{Fe}, \mathrm{Mg}$ and $\mathrm{Ni}$ ) abundances for large samples of WDs. Such large WD samples are currently being provided by SDSS, but over the next 5 years Gaia will identify $\sim 200,000$ WDs brighter than 20th magnitude within 300pc. Spectroscopic follow-up (e.g., with DESI/4MOST/WEAVE) expects to find 1000's of polluted WD (300 are known today), providing abundances for $\mathrm{Ca}$ and/or $\mathrm{Mg}$. Moreover, after Gaia DR2 (expected April, 2018), we should be able to determine the ages for a large fraction of the $>10^{4}$ WDs found to a precision of $1-2 \%$. This will help to constrain whether the accretion rates, abundance patterns or IR-excesses observed depend on the WD cooling age. Finally, after Gaia's final data release and comparative analysis of double WDs and WDs in open clusters, we should be able to derive absolute ages for a large fraction of WDs to 2\% accuracy (von Hippel et al. 2015).

Cross correlating these newly detected WDs from Gaia and the AllWISE catalogue might lead to detections of IR-excesses around these farther WDs. Follow-up of infrared excesses with JWST/MIRI will also provide direct information on the dust mineralogy that can be compared with abundances found with UV spectra. De- 
tection of the outer planetesimal belt that is feeding the accretion has been elusive because these belts are expected to be cold (Farihi et al.|2014). However, this may be possible with the potential SPICA mission (see Fig. 11 in Bonsor \& Wyatt2010). In the next 5 years, Gaia will also provide the first real insights into the population of planets around evolved stars (Silvotti et al. 2015) for which there are currently few detections so far (Xu et al. 2015). Although Gaia can only detect (greater than) Jupiter mass planets, the presence or absence of a correlation between these planets and WD pollution will constrain how WD pollution arises.

The detection of transits blocking the star-light from the polluted white dwarf WD 1145+017 has opened a new window onto the origin of WD pollution and the future detection of similar objects (possible even with relatively modest groundbased telescopes), but presumably in greater abundance with PLATO, will revolutionise our understanding of the fate of planetary systems. Continued monitoring of variability in polluted WDs (gas, dust and transits), coupled with detailed modelling, will improve our understanding of how planetary material is accreted onto WDs.

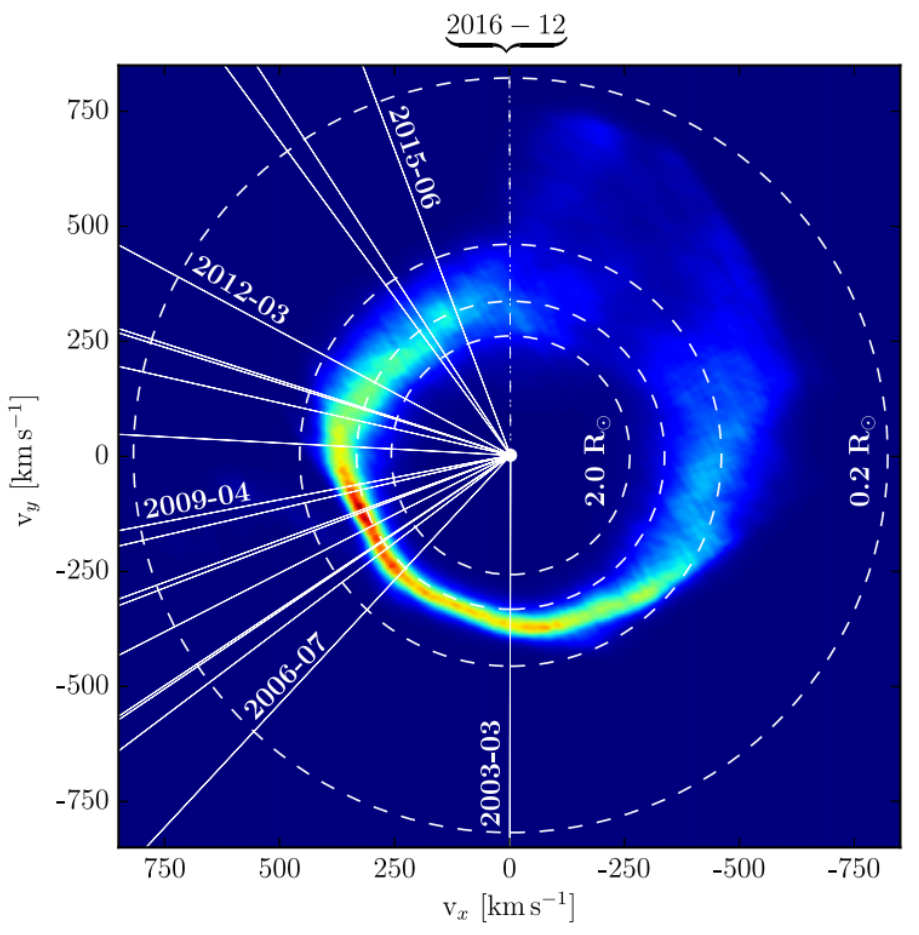

Fig. 9 An intensity distribution in velocity space of the Ca II triplet which models the line profiles observed in SDSS J1228+1040, obtained from Doppler tomography (Manser et al. 2016). 
Acknowledgements QK and MW acknowledge support from the European Union through ERC grant number 279973. CJC acknoweldges support from the DISCSIM project, grant agreement 341137 funded by the European Research Council under ERC-2013-ADG. QK thanks A. Bonsor for fruitful discussions about polluted white dwarfs.

\section{References}

Acke, B., Min, M., Dominik, C., et al. 2012, A\&A, 540, A125

Adams, F., Hollenbach, D., Laughlin,G., Gorti, U., 2004, ApJ, 611,360

Aharonian, F., Arshakian, T. G., Allen, B., et al. 2013, arXiv:1301.4124

Alexander, R., Clarke, C., Pringle, J., 2006, MNRAS, 369,229

Alexander, R. D., \& Armitage, P. J. 2007, MNRAS, 375, 500

Alexander, R., 2008, MNRAS, 391,L64

Alexander, R., Pascucci, I., Andrews, S., Armitage, P., Cieza, L., 2014, Protostars \& Planets VI, 475

Anderson, K., Adams, F., Calvet, N., 2013, ApJ, 774,9

Andrews, S. Williams, J., 2005, ApJ, 631, 1134

Andrews, S. et al, 2016 ApJ, 820, L40

Ardila, D. et al., 2013, ApJS, 207,1

Augereau, J. C., Nelson, R. P., Lagrange, A. M., Papaloizou, J. C. B., \& Mouillet, D. 2001, A\&A, 370,447

Bai, X.-N., 2013, ApJ, 772, 96

Bai, X., Stone, J, 2013, ApJ, 769,76

Balbus, S., Hawley, J., 1991, ApJ, 376,214

Benisty. M. et al, 2015, A\&A, 578,L6

Bergin, E. et al., 2013, Nature, 494,644

Beust, H., \& Morbidelli, A. 2000, Icarus, 143, 170

Beust, H., Augereau, J.-C., Bonsor, A., et al. 2014, A\&A, 561, A43

Biller, B. et al., 2013, ApJ, 777,1608

Birnstiel, T., Andrews, S., 2014, ApJ, 780, 153

Blum, J., \& Münch, M. 1993, Icarus, 106, 151

Boccaletti, A., Thalmann, C., Lagrange, A.-M., et al. 2015, Nature, 526, 230

Bonsor, A., \& Wyatt, M. 2010, MNRAS, 409, 1631

Bonsor, A., Raymond, S. N., Augereau, J.-C., \& Ormel, C. W. 2014, MNRAS, 441, 2380

Bonsor, A., Leinhardt, Z. M., Carter, P. J., et al. 2015, Icarus, 247, 291

Bonsor, A., Wyatt, M. C., Farihi, J., van Lieshout, R. . 2017, MNRAS, subm.

Booth, M., Jordán, A., Casassus, S., et al. 2016, MNRAS, 460, L10

Booth, R., Clarke, C. 2016, MNRAS, 458, 2676

Booth, R. A., Clarke, C. J, Madhusudhan, N., \& Ilee, J. D. 2017, MNRAS, subm.

Boyajian, T. S., LaCourse, D. M., Rappaport, S. A., et al. 2016, MNRAS, 457, 3988

Brandeker, A., Liseau, R., Olofsson, G., \& Fridlund, M. 2004, A\&A, 413, 681

Brandeker, A., Cataldi, G., Olofsson, G., et al. 2016, A\&A, 591, A27

Brandt, T. et al 2014, ApJ, 786,18

Bryden, G., Beichman, C. A., Carpenter, J. M., et al. 2009, ApJ, 705, 1226

Bulger, J., Patience, J., Ward-Duong, K., Pinte, C., Bouy, H., Menard, F., Monin, J.-L., 2014, A\&A, 570,29

Calvet, N., D’Alessio, P., Hartmann, L., Wilner, D., Walsh, A., Sitko, M., 2002 , ApJ, 568, 1008

Capobianco, C. C., Duncan, M., \& Levison, H. F. 2011, Icarus, 211, 819

Carilli, C. L., McKinnon, M., Ott, J., et al. 2015, arXiv:1510.06438

Carrera, D., Johansen, A., \& Davies, M. B. 2015, A\&A, 579, A43

Carrera, D., Gorti, U., Johansen, A., \& Davies, M. B. 2017, arXiv:1703.07895 
Carter-Bond, J. C., O’Brien, D. P., Delgado Mena, E., et al. 2012, ApJ, 747, L2

Carter, P. J., Leinhardt, Z. M., Elliott, T., Walter, M. J., \& Stewart, S. T. 2015, ApJ, 813, 72

Cataldi, G., Brandeker, A., Olofsson, G., et al. 2014, A\&A, 563, A66

Chiang, E., \& Youdin, A. N. 2010, Annual Review of Earth and Planetary Sciences, 38, 493

Christiaens, V., Cassasus, S., Perez, S., van der Plas, G., Menard, F., 2014 ApJ, 785, L12

Cieza, L. et al, 2013, ApJ, 762, 100

Clarke, C. 2009, MNRAS, 396,1066

Clarke, C. J., Gendrin, A., \& Sotomayor, M. 2001, MNRAS, 328, 485

Clarke, C., 2007, MNRAS, 376,1350

Clarke, C., Lodato, G., 2009, MNRAS, 398, L6

Collings, M. et al, 2004, MNRAS, 354,1133

Crida, A., Morbidelli, A., Masset, F., 2006 Icarus, 181,587

Currie, T., Kenyon, S. J., Balog, Z., et al. 2008, ApJ, 672, 558-574

Currie, T., Burrows, A., Madhusudhan, N., et al. 2013, ApJ, 776, 15

Davis, S., Stone, J., Pessah, M., 2010, ApJ, 713,52

Defrère, D., Absil, O., den Hartog, R., Hanot, C., \& Stark, C. 2010, A\&A, 509, A9

Defrère, D., Hinz, P. M., Mennesson, B., et al. 2016, ApJ, 824, 66

Dent, W. R. F., Wyatt, M. C., Roberge, A., et al. 2014, Science, 343, 1490

Dipierro, G., Price, D., Laibe, G., Hirsh, K., Cerioli, A., Lodato, G. 2015, MNRAS, 453m L730

Dong, R., Zhu, Z., Whitney, B., 2015, ApJ, 809, 93

Douglas, T. A., Caselli, P., Ilee, J. D., et al. 2013, MNRAS, 433, 2064

Drake, J., Testa, P., Hartmann, L., 2005, ApJ, 627,L149

Drovdovskaya, M., Walsh, C., Visser, R., Harsono, D., van Dishoeck, E., 2015, MNRAS, 451,3836

Durisen, R., Boss, A., Mayer, L., Nelson, A., Quinn, T., Rice, W., 2007,Protostars \& Planets V, 607

Duvert, G., Guilloteau, S., Menard, F., Simon, M., Dutrey, A., 2000, A\&A, 355, 165

Eiroa, C., Marshall, J. P., Mora, A., et al. 2013, A\&A, 555, A11

Eiroa, C., Rebollido, I., Montesinos, B., et al. 2016, A\&A, 594, L1

Ercolano, B., Clarke, C., Hall, A., 2011, MNRAS, 410,671

Ercolano, B., Owen, J., 2011, MNRAS, 406,1553

Ertel, S., Wolf, S., Marshall, J. P., et al. 2012, A\&A, 541, A148

Ertel, S., Absil, O., Defrère, D., et al. 2014, A\&A, 570, A128

Ertel, S., Augereau, J.-C., Absil, O., et al. 2015, The Messenger, 159, 24

Ertel, S., Defrère, D., Absil, O., et al. 2016, A\&A, 595, A44

Facchini, S., Clarke, C., Bisbas, T., 2016, MNRAS, 457,359

Faramaz, V., Ertel, S., Booth, M., Cuadra, J., \& Simmonds, C. 2016, arXiv:1611.02196

Farihi, J., Wyatt, M. C., Greaves, J. S., et al. 2014, MNRAS, 444, 1821

Farihi, J. 2016, New A Rev, 71, 9

Fedele, D., et al, 2010, A\&A, 510,72

Flaherty, K., Hughes, M., Rosenfeld, K., Andrews, S., Chiang, E., Simon, J., Kerzner, S., Wilner, D., 2015, ApJ, 813,99

Fridlund, C. V. M. 2004, Advances in Space Research, 34, 613

Gammie, C., 1996, ApJ, 462,725

Gammie, C. 2001, apj, 553, 174

Garufi, A. et al., 2013, A\&A, 500,105

Garufi, A., et al, 2016, A\&A, 588,8

Gáspár, A., \& Rieke, G. H. 2014, ApJ, 784, 33

Geiler, F., \& Krivov, A. 2017, arXiv:1702.05966

Gorti, U. et al, 2015, ApJ, 804,29

Grady et al, 2013, ApJ, 762, 48

Greaves, J. S., Holland, W. S., Matthews, B. C., et al. 2016, MNRAS, 461,3910

Guilloteau, S., Dutrey, A., Pietu, V., Boehler, Y., 2011, A\&A, 529,105

Guilloteau, S., di Folco, E., Dutrey, A., Simon, M., Grosso, N., Pietu, V., 2013, A\&A, 549,92

Haisch, K., Lada, E., Lada, C., 2001, ApJ, 553,L153 
Hales, A. S., Barlow, M. J., Crawford, I. A., \& Casassus, S. 2017, MNRAS, 466, 3582

Hardy, A. et al, 2015, A\&A, 583, 66

Hartmann, L, Calvet, N., Gullbring, E., D’Alessio, P., 1998, ApJ, 495, 385

Hasegawa, Y., Pudritz, R., 2013, ApJ, 778,16

Higuchi, A. E., Sato, A., Tsukagoshi, T., et al. 2017, arXiv:1703.06661

Hu, X., Zhu, Z., Tan, J., Chatterjee, S., 2016, ApJ, 816,19

Huelamo, N., Lacour, S., Tuthill, P., Ireland, M., Kraus, A., Chauvin, G., 2011, A\&A, 528,L7

Ida,S. Lin, D., 2008, ApJ, 685,584

Ilee, J. et al, 2011, MNRAS, 417,2950

Jackson, A. P., \& Wyatt, M. C. 2012, MNRAS, 425, 657

Jackson, A. P., Wyatt, M. C., Bonsor, A., \& Veras, D. 2014, MNRAS, 440, 3757

Johansen, A., Blum, J., Tanaka, H., et al. 2014, Protostars and Planets VI, 547

Johns-Krull, C. M., McLane, J. N., Prato, L., et al. 2016, ApJ, 826, 206

JOhnstone, D., Hollenbach, D., Bally, J., 1998, ApJ, 499, 758

Juhasz, A., Benisty, M., Pohl, A., Dullemond, C., Dominik, C., Paardekooper, S., 2015, MNRAS, 451,1147

Jura, M., \& Young, E. D. 2014, Annual Review of Earth and Planetary Sciences, 42, 45

Kalas, P., Graham, J. R., Fitzgerald, M. P., \& Clampin, M. 2013, ApJ, 775, 56

Kama, M., Folsom, C., Pinilla, P., 2015, A\&A, 582, L10

Kennedy, G. M., \& Wyatt, M. C. 2010, MNRAS, 405, 1253

Kennedy, G. M., \& Wyatt, M. C. 2014, MNRAS, 444, 3164

Kennedy, G. M., \& Piette, A. 2015, MNRAS, 449, 2304

Kenyon, S. J., \& Bromley, B. C. 2001, AJ, 121, 538

Kenyon, S. J., \& Bromley, B. C. 2006, AJ, 131, 1837

Kenyon, S. J., \& Bromley, B. C. 2008, ApJS, 179, 451-483

Kiefer, F., Lecavelier des Etangs, A., Augereau, J.-C., et al. 2014, A\&A, 561, L10

Kiefer, F., Lecavelier des Etangs, A., Boissier, J., et al. 2014, Nature, 514, 462

Koepferl, C., Ercolano, B., Dale, J., Teixeira, P., Ratzka, T., Spezzi, L., 2013, MNRAS, 428, 332

Koester, D., Gänsicke, B. T., \& Farihi, J. 2014, A\&A, 566, A34

Kóspál, Á., Moór, A., Juhász, A., et al. 2013, ApJ, 776, 77

Kral, Q., Thébault, P., \& Charnoz, S. 2013, A\&A, 558, A121

Kral, Q., Thébault, P., Augereau, J.-C., Boccaletti, A., \& Charnoz, S. 2015, A\&A, 573, A39

Kral, Q., Schneider, J., Kennedy, G., \& Souami, D. 2016, A\&A, 592, A39

Kral, Q., Wyatt, M., Carswell, R. F., et al. 2016, MNRAS, 461, 845

Kral, Q., \& Latter, H. 2016, MNRAS, 461, 1614

Kral, Q., Matrà, L., Wyatt, M. C., Kennedy, G. M. 2017, MNRAS, in press.

Kral, Q., Krivov, A. V., Defrere, D., et al. 2017, arXiv:1703.02540

Kraus, A., Ireland, M., 2012 ApJ, 745, 5

Krist, J. E., Ardila, D. R., Golimowski, D. A., et al. 2005, AJ, 129, 1008

Krivov, A. V., Eiroa, C., Löhne, T., et al. 2013, ApJ, 772, 32

Labeyrie, A. 2016, EAS Publications Series, 78, 45

Lacy,J., Knacke, R., Geballe, T., Tokunaga, A., 1994, ApJ, 428, 69

Lada, C., Wilking, B., 1984, ApJ, 287,610

Lambrechts, M., \& Johansen, A. 2012, A\&A, 544, A32

Le Roy, L., Altwegg, K., Balsiger, H., et al. 2015, A\&A, 583, A1

Lee, E. J., \& Chiang, E. 2016, ApJ, 827, 125

Lin, D. N. C., \& Papaloizou, J. 1979, MNRAS, 186, 799

Lissauer, J., Stevenson, D., 2007, Protostars \& Planets V, 591

Lisse, C. M., Chen, C. H., Wyatt, M. C., et al. 2009, ApJ, 701, 2019

Löhne, T., Krivov, A. V., \& Rodmann, J. 2008, ApJ, 673, 1123-1137

Madhusudhan.M., Amin, M., Kennedy, G., 2014, ApJ, 794, L12

Madhusudhan, N., Bitsch, B., Johansen, A., Ericsson, L., arXiv161103083

Manara, C. F., Rosotti, G., Testi, L., et al. 2016, A\&A, 591, L3

Manser, C. J., Gänsicke, B. T., Marsh, T. R., et al. 2016, MNRAS, 455, 4467 
Marino, S., Matrà, L., Stark, C., et al. 2016, MNRAS, 460, 2933

Marois, C., Macintosh, B., Barman, T., et al. 2008, Science, 322, 1348

Marois, C., Zuckerman, B., Konopacky, Q. M., Macintosh, B., \& Barman, T. 2010, Nature, 468, 1080

Marshall, J. P., Moro-Martín, A., Eiroa, C., et al. 2014, A\&A, 565, A15

Marshall, J. P., Cotton, D. V., Bott, K., et al. 2016, ApJ, 825, 124

Mathews, G. S., Klaassen, P. D., Juhász, A., et al. 2013, A\&A, 557, A132

Matrà, L., Panić, O., Wyatt, M. C., \& Dent, W. R. F. 2015, MNRAS, 447, 3936

Matrà, L., Dent, W. R. F., Wyatt, M. C., et al. 2017, MNRAS, 464, 1415

Matrà, L., et al. 2017, MNRAS, subm.

Matsumura, S., Pudrtiz, R., Thommes, E., 2007, ApJ, 660,1609

Matthews, B., Kennedy, G., Sibthorpe, B., et al. 2014, ApJ, 780, 97

Meng, H. Y. A., Su, K. Y. L., Rieke, G. H., et al. 2014, Science, 345, 1032

Meng, H. Y. A., Su, K. Y. L., Rieke, G. H., et al. 2015, ApJ, 805, 77

Meng, H. Y. A., Rieke, G. H., Su, K. Y. L., \& Gaspar, A. 2017, arXiv:1701.01786

Mennesson, B., Millan-Gabet, R., Serabyn, E., et al. 2014, ApJ, 797, 119

Meru, F., Bate, M., 2011, MNRAS, 411,L1

Meru, F., Bate, M., 2012, MNRAS, 427,2022

Miotello, A., Bruderer, S., van Dishoeck, E., 2014, A\&A, 572, 96

Montgomery, S. L., \& Welsh, B. Y. 2012, PASP, 124, 1042

Moór, A., Ábrahám, P., Juhász, A., et al. 2011, ApJ, 740, L7

Moór, A., Kóspál, Á., Ábrahám, P., et al. 2015, MNRAS, 447, 577

Moór, A., Henning, T., Juhász, A., et al. 2015, ApJ, 814, 42

Moro-Martín, A., Marshall, J. P., Kennedy, G., et al. 2015, ApJ, 801, 143

Mouillet, D., Larwood, J. D., Papaloizou, J. C. B., \& Lagrange, A. M. 1997, MNRAS, 292, 896

Mustill, A. J., \& Wyatt, M. C. 2009, MNRAS, 399, 1403

Muto, T. et al, 2012, ApJ, 748,L22

Najita, J., \& Williams, J. P. 2005, ApJ, 635, 625

Najita, J., Andrews, S., Muzerolle, J., 2015, MNRAS, 450,3559

Nilsson, R., Brandeker, A., Olofsson, G., et al. 2012, A\&A, 544, A134

Olofsson, G., Liseau, R., \& Brandeker, A. 2001, ApJ, 563, L77

Owen, J., 2014, ApJ, 789,59

Owen, J., Ercolano, B., Clarke, C., Alexander, R., 2010, MNRAS, 401,1415

Owen, J., CLarke, C., Ercolano, B., 2012, MNRAS, 22,1880

Owen, J., Clarke, C., 2012, MNRAS, 426,L96

Paardekooper, S.-J., Mellema, G., 2004, A\&A, 425, L9

Paardekooper, S., Baruteau, C., Meru, F. 2011, MNRAS, 416, L65

Panic, O., Hogerheijde, M., Wilner, D., Qi, C., 2009, A\&A, 501, 269

Panic, O. et al, 2013, MNRAS, 435,1037

Panic, O., Min, M., 2017, MNRAS, subm.

Pascucci, I., Herczeg, G., Carr, J., Bruderer, S., 2013, ApJ, 779, 178

Perez, L. et al, 2016, Science, 353, 1519

Perryman, M., Hartman, J., Bakos, G. Á., \& Lindegren, L. 2014, ApJ, 797, 14

Picogna, G., Kley, W., 2015, A\&A, 584,110

Pietu, V., Guilloetau, S., di Folco, E., Dutrey, A., Boehler, Y., 2014, A\&A, 564, 95

Powell, S. L., Irwin, M., Bouvier, J., \& Clarke, C. J. 2012, MNRAS, 426, 3315

Qi, C., et al., 2011, ApJ, 740,84

Qi, C. et al., 2013, Science, 341,630

Qi, C. et al., 2015, ApJ, 813,218

Quanz, S., Amara, A., Meyer, M., Kenworthy, M., Kasper, M., Girard, J., 2013, ApJ, 766,L1

Rafikov, R. 2005, ApJ, 621,L69

Rafikov, R. 2009, ApJ, 704,281

Rauer, H., Catala, C., Aerts, C., et al. 2014, Experimental Astronomy, 38, 249

Raymond, S. N., O'Brien, D. P., Morbidelli, A., \& Kaib, N. A. 2009, Icarus, 203, 644 
Raymond, S. N., Armitage, P. J., Moro-Martín, A., et al. 2012, A\&A, 541, A11

Regaly, Z., Sandor, Z., Csomos, P, Ataiee, S., 2013, MNRAS, 423, 2636

Ricci, L., Testi, L., Natta, A., Brooks, K., 2010, A\&A, 521,66

Ricci, L., Carpenter, J. M., Fu, B., et al. 2015, ApJ, 798, 124

Rice, W., Lodato, G., Pringle, J., Armitage, P, Bonnell, I., 2004, MNRAS, 355,543

Rice, W., Armitage, P., Wood, K., Lodato, G., 2006, MNRAS, 373,1619

Rice, W., Paardekooper, S., Forgan, D., Armitage, P., 2014, MNRAS, 438, 1593

Rieke, G. H., Gáspár, A., \& Ballering, N. P. 2016, ApJ, 816, 50

Roberge, A., Feldman, P. D., Lagrange, A. M., et al. 2000, ApJ, 538, 904

Roberge, A., Feldman, P. D., Weinberger, A. J., Deleuil, M., \& Bouret, J.-C. 2006, Nature, 441, 724

Roberge, A., Welsh, B. Y., Kamp, I., Weinberger, A. J., \& Grady, C. A. 2014, ApJ, 796, L11

Roelfsema, P. et al., 2014, Proc. SPIE 91431, 1K

Rosotti, G., Juhasz, A., Booth, R., Clarke, C., 2016, MNRAS, 459,2790

Rosotti, G., Booth, R., Clarke, C., Teyssandier, J., Facchini, S., Mustill, A., 2017, MNRAS, 464, L114

Sallum, S., et al., 2015, Nature, 527,342

Schneider, G., Grady, C. A., Hines, D. C., et al. 2014, AJ, 148, 59

Schoonenberg, D., \& Ormel, C. W. 2017, arXiv:1702.02151

Shannon, A., Mustill, A. J., \& Wyatt, M. 2015, MNRAS, 448, 684

Shannon, A., Bonsor, A., Kral, Q., \& Matthews, E. 2016, MNRAS, 462, L116

Silvotti, R., Sozzetti, A., Lattanzi, M., \& Morbidelli, R. 2015, 19th European Workshop on White Dwarfs, 493, 455

Simon, J., Beckwith, K., Armitage, P., 2012, MNRAS, 422,2685

Simon, J,m Bai, X.-N., Armitage, P., Stone, J., Beckwith, K., 2013, ApJ, 73, 13

Stolker, T. et al, 2016, A\&A, 595, 1135

Sullivan, P. W., Winn, J. N., Berta-Thompson, Z. K., et al. 2015, ApJ, 809, 77

Takeuchi, T., \& Artymowicz, P. 2001, ApJ, 557, 990

Tazzari, M., Testi, L., Ercolano, B., et al. 2016, A\&A, 588, A53

Teague, R., Semenove, D., Guilloteau, S., Henning, T., Dutrey, A., Wakelam, V., Chapillon, E., Pietu, V., 2015, A\&A, 574,137

Testi, L., Birnstiel, T., Ricci, L., Andrews, S., Blum, J., Carpenter, J., Dominik, C., Isella, A., Natta, A., Williams, J., Wilner, D., 2014, Protostars and Planets VI,339

Thalmann, C., et al., 2015, ApJ, 808,L41

Thébault, P., \& Wu, Y. 2008, A\&A, 481, 713

Thebault, P., Kral, Q., \& Ertel, S. 2012, A\&A, 547, A92

Thureau, N. D., Greaves, J. S., Matthews, B. C., et al. 2014, MNRAS, 445, 2558

Tobin, J. et al., 2016 Nature, 538,483

Trapman,L., Miotello, A., Kama, M., et al. 2017, A\&A, subm.

Troutman, M. R., Hinkle, K. H., Najita, J. R., Rettig, T. W., \& Brittain, S. D. 2011, ApJ, 738, 12

van Boekel, R. et al arXiv:1610.08939

van der Marel, N. et al, 2013, Science, 340,1199

van Lieshout, R., Dominik, C., Kama, M., \& Min, M. 2014, A\&A, 571, A51

von Hippel, T., van Dyk, D., Si, S., et al. 2015, 19th European Workshop on White Dwarfs, 493, 107

Vanderburg, A., Johnson, J. A., Rappaport, S., et al. 2015, Nature, 526, 546

Veras, D. 2016, Royal Society Open Science, 3, 150571

Vorobyov, E., Zakhozhay, O., Dunham, M. 2013, MNRAS, 433,3256

Wagner, K., Apai, D., Kasper, M., Robberto, M., 2015, ApJ, 813, 2

Walmswell, J., Clarke, C., \& Cossins, P. 2013, MNRAS, 431, 1903

Weidenschilling, S. J. 1977, MNRAS, 180, 57

Welsh, B. Y., \& Montgomery, S. L. 2015, Advances in Astronomy, 2015, 980323

White, J. A., Boley, A. C., Hughes, A. M., et al. 2016, ApJ, 829, 6

Williams, J., Best., W., 2014, ApJ, 788, 59 
Wilson, P. A., Lecavelier des Etangs, A., Vidal-Madjar, A., et al. 2016, arXiv:1612.00848

Wyatt, M. C., Dermott, S. F., Telesco, C. M., et al. 1999, ApJ, 527, 918

Wyatt, M. C. 2003, ApJ, 598, 1321

Wyatt, M. C. 2005, A\&A, 440, 937

Wyatt, M. C. 2006, ApJ, 639, 1153

Wyatt, M. C., Smith, R., Greaves, J. S., et al. 2007, ApJ, 658, 569

Wyatt, M. C., Smith, R., Su, K. Y. L., et al. 2007, ApJ, 663, 365

Wyatt, M. C., Kennedy, G., Sibthorpe, B., et al. 2012, MNRAS, 424, 1206

Wyatt, M. C., Panić, O., Kennedy, G. M., \& Matrà, L. 2015, Ap\&SS, 357, 103

Wyatt, M. C., Bonsor, A., Jackson, A. P., Marino, S., \& Shannon, A. 2017, MNRAS, 464, 3385

Xie, J.-W., Brandeker, A., \& Wu, Y. 2013, ApJ, 762, 114

Xu, S., Ertel, S., Wahhaj, Z., et al. 2015, A\&A, 579, L8

Young, M., Clarke, C., 2015, MNRAS, 451, 3987

Zagorovsky, K., Brandeker, A., \& Wu, Y. 2010, ApJ, 720, 923

Zhu, Z., Nelson, R., Dong, R., Espaillat, C., Hartmann, L., 2012, ApJ, 755,6

Zhu, Z., Stone, J., Rafikov, R., Bai, X., 2014, ApJ, 785, 122

Zhu, Z., Stone, J., 2017, arXiv:1701.04627

Zuckerman, B., Forveille, T., \& Kastner, J. H. 1995, Nature, 373, 494

Zuckerman, B., Koester, D., Melis, C., Hansen, B. M., \& Jura, M. 2007, ApJ, 671, 872

Zuckerman, B., \& Song, I. 2012, ApJ, 758, 77 